The Annals of Probability

2012, Vol. 40, No. 4, 1675-1714

DOI: $10.1214 / 11-A O P 662$

(C) Institute of Mathematical Statistics, 2012

\title{
A SPATIAL VERSION OF THE ITÔ-STRATONOVICH CORRECTION
}

\author{
By MARTin HAIRER ${ }^{1}$ AND JAN MAAS ${ }^{2}$ \\ University of Warwick and University of Bonn
}

\begin{abstract}
We consider a class of stochastic PDEs of Burgers type in spatial dimension 1, driven by space-time white noise. Even though it is well known that these equations are well posed, it turns out that if one performs a spatial discretization of the nonlinearity in the "wrong" way, then the sequence of approximate equations does converge to a limit, but this limit exhibits an additional correction term.

This correction term is proportional to the local quadratic crossvariation (in space) of the gradient of the conserved quantity with the solution itself. This can be understood as a consequence of the fact that for any fixed time, the law of the solution is locally equivalent to Wiener measure, where space plays the role of time. In this sense, the correction term is similar to the usual Itô-Stratonovich correction term that arises when one considers different temporal discretizations of stochastic ODEs.
\end{abstract}

1. Introduction. In this work, we give a rigorous analysis of the behavior of stochastic Burgers equations in one spatial dimension under various approximation schemes. It was recently argued in [12] that if the approximation scheme fails to satisfy a certain symmetry condition, then one expects the approximations to converge to a modified equation, with the appearance of an additional correction term in the limit. This correction term is somewhat similar to the Itô-Stratonovich correction that appears in the study of SDEs when one compares centred and one-sided approximations. The present article provides a rigorous justification of the results observed in [12], at least

Received November 2010; revised March 2011.

${ }^{1}$ Supported by the EPSRC Grants EP/E002269/1 and EP/D071593/1, a Wolfson Research Merit Award of the Royal Society and a Philip Leverhulme prize of the Leverhulme Trust.

${ }^{2}$ Supported by Rubicon Grant 680-50-0901 of the Netherlands Organisation for Scientific Research (NWO).

AMS 2000 subject classifications. Primary 60H15; secondary 35K55, 60H30, 60H35.

Key words and phrases. Itô-Stratonovich correction, stochastic Burgers equation, spatial discretizations, Wiener chaos.

This is an electronic reprint of the original article published by the Institute of Mathematical Statistics in The Annals of Probability, 2012, Vol. 40, No. 4, 1675-1714. This reprint differs from the original in pagination and typographic detail. 
in the case where the nonlinearity of the equation is of gradient type, and therefore the limiting equation is well posed in a classical sense.

More precisely, we will consider in this work stochastic PDEs of the form

$$
\partial_{t} u(x, t)=\nu \partial_{x}^{2} u(x, t)+F(u(x, t))+(\nabla G)(u(x, t)) \partial_{x} u(x, t)+\xi(x, t),
$$

where $u=u(x, t)$ is an $\mathbf{R}^{n}$-valued function, with $x \in[0,2 \pi], t \geq 0$. In this equation, $\nu>0$ is a positive constant, the functions $F, G: \mathbf{R}^{n} \rightarrow \mathbf{R}^{n}$ are assumed to be $\mathcal{C}^{3}$, and the stochastic forcing term $\xi$ consists of independent space-time white noises in each component of $\mathbf{R}^{n}$. For the sake of simplicity, we endow this equation with periodic boundary conditions, but we do not expect this to alter our results significantly.

Endowed with an initial condition $u_{0} \in \mathcal{C}\left([0,2 \pi] ; \mathbf{R}^{n}\right),(1.1)$ is locally well posed [9], provided that we rewrite the nonlinearity as $\partial_{x} G(u)$ and consider solutions either in the weak or the mild form [7]. (Note that our noise term is not the gradient of space-time white noise, as in [3]. Therefore, our solutions are actually $\alpha$-Hölder continuous functions for all $\alpha<\frac{1}{2}$.) The aim of this article is to show that this well-posedness is much less stable than one may imagine at first. Indeed, if we set

$$
D_{\varepsilon}^{+} u(x)=\frac{u(x+\varepsilon)-u(x)}{\varepsilon},
$$

and consider the family $u_{\varepsilon}$ of solutions to the approximate equation

$$
\partial_{t} u_{\varepsilon}=\nu \partial_{x}^{2} u_{\varepsilon}+F\left(u_{\varepsilon}\right)+\nabla G\left(u_{\varepsilon}\right) D_{\varepsilon}^{+} u_{\varepsilon}+\xi,
$$

then our main result, Theorem 1.6 below, implies that $u_{\varepsilon} \Rightarrow \bar{u}$, where $\bar{u}$ is the solution to (1.1), but with $F$ replaced by

$$
\bar{F}(u)=F(u)-\frac{1}{4 \nu} \Delta G(u),
$$

where $\Delta$ is the usual Laplacian on $\mathbf{R}^{n}$.

REMARK 1.1. The correction term in (1.2) is reminiscent of the WongZakai correction [17], which arises if the driving Brownian motion in a stochastic ODE or PDE is approximated by stochastic processes of bounded variation. This correction term is due to the temporal roughness of the driving Brownian motion and does not appear if the noise is additive.

Our correction term is a consequence of the spatial roughness of the solutions and appears even though we consider SPDEs with additive noise. In fact, an explicit calculation allows to check that the local quadratic variation (in space) of the solution $u$ to (1.1) is precisely given by $1 /(2 \nu)$. Therefore, one can interpret the correction term appearing in (1.2) as precisely being equal to $-\frac{1}{2}$ times the quadratic covariation between $u$ and $\nabla G(u)$. Recall that this is exactly the correction term that appears when one switches between Itô and Stratonovich integral in the usual setting of stochastic calculus. See also [12] for a heuristic argument for computing the correction term. 
REMARK 1.2. This correction term is a purely stochastic effect and is completely unrelated to the fact that our discretization scheme is not an upwind scheme (see $[5,15])$. In the absence of noise, we would still have the regularizing property from the nonvanishing viscosity, so that pretty much any "reasonable" numerical scheme would converge to the correct solution.

If $D_{\varepsilon}^{+}$is replaced by $D_{\varepsilon}^{-}$, defined by $D_{\varepsilon}^{-} u(x)=(u(x)-u(x-\varepsilon)) / \varepsilon$, then a similar result is true, but the sign in front of the correction term in (1.2) changes. We will actually consider a much more general class of approximations to (1.1), where we also allow both the linear operator $\partial_{x}^{2}$ and the noise term $\xi$ to be replaced by approximate versions that are still translationinvariant, but modified at the lengthscale $\varepsilon$.

1.1. Statement of the main result. For $\varepsilon>0$, we consider approximating stochastic PDEs of the type

$$
\partial_{t} u_{\varepsilon}=\nu \Delta_{\varepsilon} u_{\varepsilon}+F\left(u_{\varepsilon}\right)+\nabla G\left(u_{\varepsilon}\right) D_{\varepsilon} u_{\varepsilon}+\xi_{\varepsilon} .
$$

Since our system is invariant under spatial translations, it seems natural to restrict ourselves to a class of approximations that enjoys the same property. Throughout this article, we will therefore use approximate differential operators $\Delta_{\varepsilon}$ and $D_{\varepsilon}$, as well as an approximate space-time white noise $\xi_{\varepsilon}$ given by their Fourier transforms:

$$
\begin{aligned}
\widehat{\Delta_{\varepsilon} u}(k) & =-k^{2} f(\varepsilon|k|) \widehat{u}(k), \quad \widehat{D_{\varepsilon} u}(k)=i k g(\varepsilon k) \widehat{u}(k), \\
\widehat{\xi}_{\varepsilon}(k) & =h(\varepsilon|k|) \widehat{\xi}(k) .
\end{aligned}
$$

Several natural discretizations arising in numerical analysis are of this form (see the examples below Theorem 1.6). We will make the following standing assumptions on these objects.

Assumption 1.3. The function $f:[0, \infty) \rightarrow[0,+\infty]$ is twice differentiable at 0 with $f(0)=1$ and $f^{\prime}(0)=0$. Furthermore, there exists $q \in(0,1]$ such that $f(k) \geq q$ for all $k>0$.

If $f(k)=+\infty$ for some values of $k$, we use the convention $\exp (-t \infty)=0$ for every $t>0$. In this case, the semigroup generated by $\Delta_{\varepsilon}$ is not strongly continuous, but this is of no consequence for our analysis.

Assumption 1.4. There exists a signed Borel measure $\mu$ such that

$$
\int_{\mathbf{R}} e^{i k x} \mu(d x)=i k g(k),
$$

and such that

$$
\mu(\mathbf{R})=0, \quad|\mu|(\mathbf{R})<\infty, \int_{\mathbf{R}} x \mu(d x)=1, \quad \int_{\mathbf{R}}|x|^{4}|\mu|(d x)<\infty .
$$

In particular, we have $\left(D_{\varepsilon} u\right)(x):=\frac{1}{\varepsilon} \int_{\mathbf{R}} u(x+\varepsilon y) \mu(d y)$. 
Assumption 1.5. The function $h$ is bounded and such that $h^{2} / f$ is of bounded variation. Furthermore, $h$ is twice differentiable at the origin with $h(0)=1$ and $h^{\prime}(0)=0$.

Let $\bar{u}$ be the solution to the equation

$$
\partial_{t} \bar{u}=\nu \partial_{x}^{2} \bar{u}+\bar{F}(\bar{u})+\nabla G(\bar{u}) \partial_{x} \bar{u}+\xi .
$$

In this equation,

$$
\bar{F}:=(F-\Lambda \Delta G)
$$

and $\Lambda \in \mathbf{R}$ is a correction constant given by

$$
\Lambda \stackrel{\text { def }}{=} \frac{1}{2 \pi \nu} \int_{\mathbf{R}_{+}} \int_{\mathbf{R}} \frac{(1-\cos (y t)) h^{2}(t)}{t^{2} f(t)} \mu(d y) d t .
$$

Note that a straightforward calculation shows that $\Lambda$ is indeed well defined, as a consequence of the fact that $h^{2} / f$ is bounded by assumption and that $|\mu|$ has a finite second moment.

Before we state our main result, note that the equation (1.4) is locally well posed in $L^{\infty}$, see $[2,4,6,9,10]$. As a consequence, it has a well-defined blowup time $\tau_{*}$ (possibly infinite) such that, almost surely, $\lim _{t \rightarrow \tau_{*}}\|\bar{u}(t)\|_{L^{\infty}}=$ $+\infty$ on the event $\left\{\tau_{*}<\infty\right\}$. With this notation, we are now ready to state the main result of this paper.

TheOREM 1.6. Let $\kappa>0$ and let $u_{\varepsilon}$ and $\bar{u}$ have initial conditions as in Theorem 2.2. There exists a sequence of stopping times $\tau_{\varepsilon}$ satisfying $\lim _{\varepsilon \rightarrow 0} \tau_{\varepsilon}=\tau_{*}$ in probability, and such that

$$
\lim _{\varepsilon \rightarrow 0} \mathbb{P}\left(\sup _{t \leq \tau_{\varepsilon}}\left\|u_{\varepsilon}(t)-\bar{u}(t)\right\|_{L^{\infty}}>\varepsilon^{1 / 8-\kappa}\right)=0 .
$$

REMARK 1.7. In order to avoid further technical complications, we consider sequences of initial conditions that have the property that the initial condition for $u_{\varepsilon}$ "behaves like" the solution $u_{\varepsilon}(t)$ for positive times. In fact, the initial condition for $u_{\varepsilon}$ is a smooth perturbation of the stationary solution to the linearized equation for $u_{\varepsilon}$. We refer to Section 2 for more details.

Before we proceed, we list some of the most common examples of discretizations that do fit our framework. For $a, b \geq 0$ with $a+b>0$, it is natural to discretize the derivative operator by choosing

$$
\mu:=\frac{\delta_{a}-\delta_{-b}}{a+b} .
$$

This is also the discretization that was considered in [12]. As far as the discretizations of the noise and the Laplacian are concerned, there are at least three natural choices. 
No discretization. This is the case $f=h=1$ where only the nonlinearity is discretized. With this choice, one can check that the correction factor is given by $\Lambda=\frac{1}{4 \nu} \frac{a-b}{a+b}$.

Finite difference discretization. In this case, we divide the interval $[0,2 \pi]$ into $N$ equally sized intervals. For convenience, we assume that $N$ is odd and we set

$$
\left(\Delta_{\varepsilon} u\right)(x)=\frac{1}{\varepsilon^{2}}(u(x+\varepsilon)+u(x-\varepsilon)-2 u(x)), \quad \varepsilon=\frac{2 \pi}{N} .
$$

We furthermore identify a function $u$ with the trigonometric polynomial of degree $(N-1) / 2$ agreeing with $u$ at the gridpoints. This corresponds to the choice

$$
f(k)= \begin{cases}\frac{4}{k^{2}} \sin ^{2}(k / 2), & k \in[0, \pi), \\ +\infty, & k \in[\pi, \infty),\end{cases}
$$

The natural choice for the discretization of the derivative operator in this case is to choose $a$ and $b$ to be integers, so that discretization takes place on the gridpoints. With this choice, it can be shown that the correction factor is identical to that obtained in the previous case. Note however that this is not the case if the discretization of the derivative operator is not adapted to the gridsize.

Galerkin discretization. In this case, we approximate $\Delta$ and $\xi$ by only keeping those Fourier modes that appear in the approximation by trigonometric polynomials. This corresponds to the choice

$$
f(k)=\left\{\begin{array}{ll}
1, & k \in[0, \pi), \\
+\infty, & k \in[\pi, \infty),
\end{array} \quad h=\mathbf{1}_{[0, \pi)} .\right.
$$

The correction factor $\Lambda$ is then given by

$$
\Lambda=\frac{\cos (\pi a)+\pi a \operatorname{Si}(\pi a)-\cos (\pi b)-\pi b \operatorname{Si}(\pi b)}{2 \pi^{2} \nu(a+b)},
$$

where Si $t=\int_{0}^{t} \frac{\sin x}{x} d x$.

The rest of this paper is structured as follows. In Section 2, we introduce notation, we give a refined formulation of the main result and present an outline of the proof of the main result (Theorem 2.2). In Section 3, we prove several useful bounds on the approximating semigroups and the approximations of the gradient. Section 4 is devoted to several estimates for stochastic convolutions, the most crucial one being Proposition 4.6, which is responsible for the correction term appearing in the limiting equation. Most of the work is performed in Section 5, where convergence of various approximating equations is proved. 
2. Proof of the main result. In order to shorten notation, we introduce the semigroups $S$ and $S_{\varepsilon}$, defined as rescaled versions of the heat semigroup and its approximation:

$$
S(t) \stackrel{\text { def }}{=} e^{-t\left(1-\nu \partial_{x}^{2}\right)}, \quad S_{\varepsilon}(t) \stackrel{\text { def }}{=} e^{-t\left(1-\nu \Delta_{\varepsilon}\right)},
$$

where we define $S_{\varepsilon}$ by Fourier analysis, that is,

$$
\widehat{S_{\varepsilon} u}(k)=e^{-t\left(1+\nu k^{2} f(\varepsilon|k|)\right)} \widehat{u}(k),
$$

making use of the convention $e^{-\infty}=0$.

Since we will always work with the mild formulation, it will be convenient to have a notation for the convolution (in time) of a function with one of the semigroups. We will henceforth write

$$
(S * w)(t) \stackrel{\text { def }}{=} \int_{0}^{t} S(t-s) w(s) d s .
$$

Let $(W(t))_{t \in \mathbf{R}}$ be a two-sided cylindrical Wiener process on $\mathcal{H} \stackrel{\text { def }}{=} L^{2}([0,2 \pi]$, $\mathbf{R}^{n}$ ) (see $[7,10]$ for precise definitions) and let $Q_{\varepsilon}$ be the bounded operator on $\mathcal{H}$ defined as a Fourier multiplier by

$$
\widehat{Q_{\varepsilon} u}(k)=h(\varepsilon|k|) \widehat{u}(k) .
$$

(We assume that it acts independently on each component.) Finally, we define the $\mathcal{H}$-valued processes $\psi$ and $\widetilde{\psi}$ by

$$
\psi(t)=\int_{-\infty}^{t} S(t-s) d W(s), \quad \widetilde{\psi}(t)=\int_{-\infty}^{t} S_{\varepsilon}(t-s) Q_{\varepsilon} d W(s),
$$

so that, in the notation of the previous section, they are the stationary solutions to the linear equations

$$
\partial_{t} \psi=\left(\nu \partial_{x}^{2}-1\right) \psi+\xi, \quad \partial_{t} \widetilde{\psi}=\left(\nu \Delta_{\varepsilon}-1\right) \widetilde{\psi}+\xi_{\varepsilon} .
$$

With this notation at hand, we can rewrite the equations for $\bar{u}$ and $u_{\varepsilon}$ in the mild form as

$$
\begin{aligned}
\bar{u}(t) & =S(t) v_{0}+\psi(t)+S *\left(\bar{F}(\bar{u})+\nabla G(\bar{u}) \partial_{x} \bar{u}\right)(t), \\
u_{\varepsilon}(t) & =S_{\varepsilon}(t) v_{0}+\widetilde{\psi}(t)+S_{\varepsilon} *\left(F\left(u_{\varepsilon}\right)+\nabla G\left(u_{\varepsilon}\right) D_{\varepsilon} u_{\varepsilon}\right)(t) .
\end{aligned}
$$

REMARK 2.1. Note that we have used here a common initial condition $v_{0}$ for the difference $\bar{u}-\psi$ and $u_{\varepsilon}-\widetilde{\psi}$. As a consequence, the two equations do not start with the same initial condition! However, as $\varepsilon \rightarrow 0$, the initial condition of $u_{\varepsilon}$ converges to that of $\bar{u}$. The reason for not starting with the same initial condition is mostly of technical nature.

It will be convenient to define for any $0<\gamma<\chi$,

$$
\psi_{\gamma} \stackrel{\text { def }}{=}\left(I-\Pi_{\varepsilon^{-\gamma}}\right) \psi, \quad \psi^{\gamma} \stackrel{\text { def }}{=} \Pi_{\varepsilon^{-\gamma}} \psi, \quad \psi_{\gamma}^{\chi} \stackrel{\text { def }}{=}\left(\Pi_{\varepsilon^{-\chi}}-\Pi_{\varepsilon^{-\gamma}}\right) \psi .
$$


The expressions $\widetilde{\psi}_{\gamma}, \widetilde{\psi}^{\gamma}$ and $\widetilde{\psi}_{\gamma}^{\chi}$ are defined analogously. Here $\Pi_{N}$ denotes the projection onto the low-frequency components of the Fourier expansion, defined by $\Pi_{N} e_{n} \stackrel{\text { def }}{=} \mathbf{1}_{|n| \leq N} e_{n}$, where $e_{n}(x)=(2 \pi)^{-1 / 2} e^{i n x}$.

We set

$$
\bar{v} \stackrel{\text { def }}{=} \bar{u}-\psi, \quad \widetilde{v} \stackrel{\text { def }}{=} u_{\varepsilon}-\widetilde{\psi} .
$$

In the proof, it will be convenient to work with the functions $\bar{v}^{\gamma}$ and $\widetilde{v}^{\gamma}$ defined by

$$
\bar{v}^{\gamma} \stackrel{\text { def }}{=} \bar{v}+\psi^{\gamma}=\bar{u}-\psi_{\gamma}, \quad \widetilde{v}^{\gamma} \stackrel{\text { def }}{=} \widetilde{v}+\widetilde{\psi}^{\gamma}=u_{\varepsilon}-\widetilde{\psi}_{\gamma} .
$$

It follows from (2.1) and (2.2) that these functions satisfy the following equations:

$$
\begin{aligned}
\bar{v}^{\gamma}(t)= & S(t) v_{0}+\psi^{\gamma}(t)+S *\left(\bar{F}\left(\bar{v}^{\gamma}+\psi_{\gamma}\right)+\partial_{x}\left(G\left(\bar{v}^{\gamma}+\psi_{\gamma}\right)\right)\right)(t), \\
\widetilde{v}^{\gamma}(t)= & S_{\varepsilon}(t) v_{0}+\widetilde{\psi}^{\gamma}(t) \\
& +S_{\varepsilon} *\left(F\left(\widetilde{v}^{\gamma}+\widetilde{\psi}_{\gamma}\right)+\nabla G\left(\widetilde{v}^{\gamma}+\widetilde{\psi}_{\gamma}\right) D_{\varepsilon}\left(\widetilde{v}^{\gamma}+\widetilde{\psi}_{\gamma}\right)\right)(t) .
\end{aligned}
$$

For large parts of this article, it will be convenient to work in the fractional Sobolev space $H^{\alpha}$ for some $\alpha>\frac{1}{2}$, so that $H^{\alpha} \subset L^{\infty}$. Recall that $H^{\alpha}$ denotes the space of (equivalence classes of) functions $u=\sum_{j \in \mathbf{Z}} u_{k} e_{k}$ on $[0,2 \pi]$ with $u_{k} \in \mathbf{C}$, for which

$$
\|u\|_{\alpha}^{2}:=\sum_{k \in \mathbf{Z}}\left|u_{k}\right|^{2}\left(1+k^{2}\right)^{\alpha}<\infty .
$$

Furthermore, we will need to use a high-frequency cut-off, which will smoothen out the solutions at a scale $\varepsilon^{\chi}$ for some $\chi>1$. It turns out that a reasonable choice for these parameters is given by

$$
\alpha=\frac{3}{4}, \quad \gamma=\frac{1}{3}, \quad \chi=\frac{3}{2},
$$

and we will fix these values from now on. With this notation at hand, the following theorem, which is essentially a more precise reformulation of Theorem 1.6, is a more precise statement of our main result. Here and in the rest of the paper we write $\|u\|_{\beta}$ to denote the norm of an element $u$ in the fractional Sobolev space $H^{\beta}$ for $\beta \in \mathbf{R}$.

TheOREM 2.2. Let $\kappa>0$ be an arbitrary (small) exponent and let $v_{0} \in H^{\beta}$ for all $\beta<\frac{3}{2}$. There exists a sequence of stopping times $\tau_{\varepsilon}$ satisfying $\tau_{\varepsilon} \rightarrow \tau_{*}$ in probability as $\varepsilon \rightarrow 0$, such that

$$
\lim _{\varepsilon \rightarrow 0} \mathbb{P}\left(\sup _{t \leq \tau_{\varepsilon}}\left\|u_{\varepsilon}(t)-\bar{u}(t)\right\|_{L^{\infty}}>\varepsilon^{1 / 8-\kappa}\right)=0 .
$$


In fact, we have the bounds

$$
\begin{aligned}
\lim _{\varepsilon \rightarrow 0} \mathbb{P}\left(\sup _{t \leq \tau_{\varepsilon}}\left\|\widetilde{v}^{\gamma}(t)-\bar{v}^{\gamma}(t)\right\|_{\alpha}>\varepsilon^{1 / 8-\kappa}\right) & =0, \\
\lim _{\varepsilon \rightarrow 0} \mathbb{P}\left(\sup _{t \leq \tau_{\varepsilon}}\left\|\widetilde{\psi}_{\gamma}(t)-\psi_{\gamma}(t)\right\|_{L^{\infty}}>\varepsilon^{1 / 2-\kappa}\right) & =0 .
\end{aligned}
$$

REMARK 2.3. We emphasize again that the initial conditions $\bar{u}(0)$ and $u_{\varepsilon}(0)$ are slightly different. In fact, one has $u_{\varepsilon}(0)=\bar{u}(0)+\widetilde{\psi}(0)-\psi(0)$.

REMARK 2.4. The rate $\frac{1}{8}$ is not optimal. By adjusting the parameters $\alpha$, $\gamma$ and $\chi$ in an optimal way, and by sharpening some of the arguments in our proof, one could achieve a slightly better rate. However, we do not believe that any rate obtained in this way would reflect the true speed of convergence, so we keep with the values (2.5) that yield simple fractions.

REMARK 2.5. From a technical point of view, the general methodology followed in this section and the subsequent sections is inspired from [11], where a somewhat similar phenomenon was investigated. Besides the structural differences in the equations considered here and in [11], the main technical difficulties that need to be overcome for the present work are the following:

(1) In [11], it is possible to simply subtract the stochastic convolution $\psi$ (or $\widetilde{\psi}$ ) and work with the equation for the remainder. Here, we instead subtract only the highest Fourier modes of $\psi$. The reason for this choice is that it entails that $\bar{v}^{\gamma} \rightarrow \bar{u}$ as $\varepsilon \rightarrow 0$. This allows us to linearize the nonlinearity around $\bar{v}^{\gamma}$ in order to exhibit the desired correction term. As a consequence, our a priori regularity estimates are much worse than those in [11] and our convergence rates are worse. The main reason why we need this complication is that our approximate derivative $D_{\varepsilon}$ does not satisfy the chain rule.

(2) All of our fixpoint arguments need to be performed in the fractional Sobolev space $H^{\alpha}$, for some $\alpha>\frac{1}{2}$. This is in contrast to [11] where some of the arguments could be performed first in $L^{\infty}$, and then lifted to $H^{\alpha}$ by a standard bootstrapping argument. These bootstrapping arguments fail here, since the nonlinearity of our approximating equation contains an approximate derivative, which gives rise to correction terms which are not easy to control.

(3) In one crucial step where a Gaussian concentration inequality is employed in [11], it was necessary that the stochastic convolutions belong to $H^{\alpha}$ for some $\alpha>\frac{1}{2}$. This is the case in [11] as a consequence of the extra regularizing effect caused by a small fourth-order term present in the linear part. This additional regularizing effect is not always present in the current work. We therefore perform another truncation in Fourier space, at very high frequencies. This is the purpose of the exponent $\chi$. 
Note also that Proposition 4.6 is the analogue of Proposition 4.1 in [11]. One difference is that we have a much cleaner separation of the probabilistic and the analytical aspects of this result.

By a standard Picard fixed point argument (see, e.g., [10]) it can be shown that (2.1) admits a unique mild solution $\bar{u}$ defined on a random time interval $\left[0, \tau_{*}\right]$. Moreover, the spatial regularity of $\psi$ and $\bar{u}$ equals that of a Brownian path, in the sense that $\psi(t)$ and $\bar{u}(t)$ are continuous and belong to $H^{\beta}$ for any $\beta<\frac{1}{2}$ and any $t>0$, but not to $H^{1 / 2}$. We shall take advantage of the fact that the process $\bar{v}$ is much more regular. In fact, $\bar{v}(t) \in H^{\beta}$ almost surely for any $\beta<\frac{3}{2}$ and any $t>0$, but one does not expect it to belong to $H^{3 / 2}$ in general. This follows immediately from the mild formulation (2.1) combined with a standard bootstrapping argument. It follows from these considerations that, for every fixed time horizon $T$, the stopping time

$$
\tau_{*}^{K}:=T \wedge \inf \left\{t:\|\bar{v}(t)\|_{\alpha} \vee\|\bar{u}(t)\|_{L^{\infty}} \geq K\right\}
$$

converges in probability to $\tau_{*} \wedge T$ as $K \rightarrow \infty$.

It will be shown in Section 4 that a number of functionals of $\psi$ and $\widetilde{\psi}$ scale in the following way:

$$
\begin{aligned}
&\left\|\widetilde{\psi}_{\gamma}^{\chi}(t)\right\|_{L^{\infty}} \lesssim \varepsilon^{\gamma / 2-\kappa}, \quad\left\|\widetilde{\psi}_{\gamma}(t)\right\|_{L^{\infty}} \lesssim \varepsilon^{\gamma / 2-\kappa}, \\
&\left\|\psi_{\gamma}(t)\right\|_{L^{\infty}} \lesssim \varepsilon^{\gamma / 2-\kappa}, \quad\left\|\widetilde{\psi}_{\chi}(t)\right\|_{L^{\infty}} \lesssim \varepsilon^{\chi / 2-\kappa}, \\
&\left\|\psi^{\gamma}(t)\right\|_{\alpha} \lesssim \varepsilon^{-\gamma(\alpha-1 / 2)-\kappa}, \quad\left\|\widetilde{\psi}_{\gamma}^{\chi}(t)\right\|_{\alpha} \lesssim \varepsilon^{-\chi(\alpha-1 / 2)-\kappa}, \\
&\left\|\widetilde{\psi}^{\gamma}(t)-\psi^{\gamma}(t)\right\|_{\alpha} \lesssim \varepsilon^{2-\gamma(\alpha+3 / 2)-\kappa}, \quad \Theta_{\varepsilon}\left(\widetilde{\psi}_{\gamma}(t)\right) \lesssim \varepsilon^{-1-\kappa}, \\
& \Theta_{\varepsilon}\left(\widetilde{\psi}_{\chi}(t)\right) \lesssim \varepsilon^{\chi-2-\kappa}, \quad\left\|\Lambda-\Xi_{\varepsilon}\left(\widetilde{\psi}_{\gamma}^{\chi}(t)\right)\right\|_{-\alpha} \lesssim \varepsilon^{1 / 2-\kappa},
\end{aligned}
$$

where the quantities $\Theta_{\varepsilon}$ and $\Xi_{\varepsilon}$ are defined by

$$
\Theta_{\varepsilon}(u) \stackrel{\text { def }}{=} \int_{\mathbf{R}} y^{2}\left\|\widehat{D}_{\varepsilon y} u\right\|_{L^{2}}^{2}|\mu|(d y), \quad \Xi_{\varepsilon}(u) \stackrel{\text { def }}{=} \int_{\mathbf{R}} \frac{\varepsilon y^{2}}{2} \widehat{D}_{\varepsilon y} u \otimes \widehat{D}_{\varepsilon y} u \mu(d y) .
$$

Note that all of these relations are of the form $\Psi_{i}^{\varepsilon}(t) \lesssim \varepsilon^{\alpha_{i}-\kappa}$ for some expression $\boldsymbol{\Psi}_{i}^{\varepsilon}$ depending on $\varepsilon$ and some exponent $\alpha_{i}$. In the proof, it will be convenient to impose this behavior by means of a hard constraint. For this purpose, we introduce the stopping time $\tau^{K}$, which is defined for $K>0$ by

$$
\tau^{K} \stackrel{\text { def }}{=} \tau_{*}^{K} \wedge \inf \left\{t: \exists i: \Psi_{i}^{\varepsilon}(t) \geq \varepsilon^{\alpha_{i}-\kappa}\right\} .
$$

From now on, we will write $C_{K}$ to denote a constant which may depend on $K$ (and $T$ ) and is allowed to change from line to line. Similarly, $\kappa$ will be a positive universal constant which is sufficiently small and whose value is allowed to change from line to line. However, the final value of $\kappa$ is independent of $\varepsilon$, $K$ and $T$.

The remainder of this section is devoted to the proof of Theorem 2.2. 
Proof OF TheOrem 2.2. Most of the work in the proof consists of bounding the difference between $\widetilde{v}^{\gamma}$ and $\bar{v}^{\gamma}$ in $H^{\alpha}$. This bound will be obtained in several steps, using the intermediate processes $v_{\varepsilon}^{(i)}, i=1, \ldots, 4$, defined by

$$
\begin{aligned}
v_{\varepsilon}^{(1)}(t)= & S(t) v_{0}+\psi^{\gamma}(t)+S *\left(\bar{F}\left(v_{\varepsilon}^{(1)}\right)+\partial_{x} G\left(v_{\varepsilon}^{(1)}\right)\right)(t), \\
v_{\varepsilon}^{(2)}(t)= & S(t) v_{0}+\psi^{\gamma}(t)+S *\left(\bar{F}\left(v_{\varepsilon}^{(2)}\right)+D_{\varepsilon} G\left(v_{\varepsilon}^{(2)}\right)\right)(t), \\
v_{\varepsilon}^{(3)}(t)= & S_{\varepsilon}(t) v_{0}+\widetilde{\psi}^{\gamma}(t)+S_{\varepsilon} *\left(\bar{F}\left(v_{\varepsilon}^{(3)}\right)+D_{\varepsilon} G\left(v_{\varepsilon}^{(3)}\right)\right)(t), \\
v_{\varepsilon}^{(4)}(t)= & S_{\varepsilon}(t) v_{0}+\widetilde{\psi}^{\gamma}(t) \\
& +S_{\varepsilon} *\left(F\left(v_{\varepsilon}^{(4)}+\widetilde{\psi}_{\gamma}^{\chi}\right)+\nabla G\left(v_{\varepsilon}^{(4)}+\widetilde{\psi}_{\gamma}^{\chi}\right) D_{\varepsilon}\left(v_{\varepsilon}^{(4)}+\widetilde{\psi}_{\gamma}^{\chi}\right)\right)(t) .
\end{aligned}
$$

At this stage, we stress that the main difficulty of the proof consists of showing that $v_{\varepsilon}^{(3)}$ and $v_{\varepsilon}^{(4)}$ are close (see Proposition 5.5 below). Showing the smallness of the remaining differences $v_{\varepsilon}^{(j)}-v_{\varepsilon}^{(j+1)}$ is relatively straightforward and follows by applying standard SPDE techniques. The main ingredient in this part of the proof is an estimate which compares the square of the approximate derivative of $\widetilde{\psi}_{\gamma}^{\chi}$ to the correction term, in a suitable Sobolev space of negative order. The estimate is purely probabilistic and ultimately relies on the fact that the quantity that we wish to control belongs to the second order Wiener chaos. It can be found in Proposition 4.6, which we consider to be the core of the paper.

Recall the definition of the stopping time $\tau^{K}$ given in (2.9). With this definition at hand, we set $\tau_{0}^{K} \stackrel{\text { def }}{=} \tau^{K}$ as well as $v_{\varepsilon}^{(0)} \stackrel{\text { def }}{=} \bar{v}^{\gamma}$ and $v_{\varepsilon}^{(5)} \stackrel{\text { def }}{=} \widetilde{v}^{\gamma}$, and we define recursively a sequence of stopping times $\tau_{j}^{K}$ with $j=1, \ldots, 5$ by

$$
\tau_{j}^{K}=\tau_{j-1}^{K} \wedge \inf \left\{t:\left\|v_{\varepsilon}^{(j)}(t)-v_{\varepsilon}^{(j-1)}(t)\right\|_{\alpha} \geq K\right\} .
$$

With this notation at hand, Propositions 5.1-5.7 state that, for all fixed values $K, \kappa>0$ and every $j=1, \ldots, 5$, one has

$$
\lim _{\varepsilon \rightarrow 0} \mathbb{P}\left(\sup _{t \leq \tau_{j}^{K}}\left\|v_{\varepsilon}^{(j)}(t)-v_{\varepsilon}^{(j-1)}(t)\right\|_{\alpha}>\varepsilon^{1 / 8-\kappa}\right)=0 .
$$

Combining all of these bounds, we conclude immediately that, for every fixed time horizon $T>0$ and every choice of values $K$ and $\kappa$, we have

$$
\lim _{\varepsilon \rightarrow 0} \mathbb{P}\left(\sup _{t \leq \tau_{5}^{K}}\left\|\widetilde{v}^{\gamma}(t)-\bar{v}^{\gamma}(t)\right\|_{\alpha}>\varepsilon^{1 / 8-\kappa}\right)=0 .
$$

This is formally very close to (2.7), except that we still have the values $T, K>0$ appearing in our statement and consider the solutions only up to the stopping time $\tau_{5}^{K}$.

Since $\tau_{*} \wedge T \rightarrow \tau_{*}$ as $T \rightarrow \infty$ and since we already argued that $\tau_{*}^{K} \rightarrow \tau_{*} \wedge T$ as $K \rightarrow \infty$, the bound (2.7) follows if we are able to show that, for every 
fixed choice of $K$ and $T$,

$$
\lim _{\varepsilon \rightarrow 0} \mathbb{P}\left(\tau_{5}^{K} \neq \tau_{*}^{K}\right)=0 .
$$

Since the statement of our theorem is stronger, the smaller the value of $\kappa$, we can assume without loss of generality that $\kappa<\frac{1}{8}$. In this case, $\lim _{\varepsilon \rightarrow 0} \varepsilon^{1 / 8-\kappa}=$ 0 , so that (2.12) and (2.11) together imply that

$$
\lim _{\varepsilon \rightarrow 0} \mathbb{P}\left(\tau_{j}^{K} \neq \tau_{j-1}^{K}\right)=0
$$

for $j=1, \ldots, 5$, from which we conclude that $\lim _{\varepsilon \rightarrow 0} \mathbb{P}\left(\tau_{5}^{K} \neq \tau^{K}\right)=0$.

In order to finish the proof of (2.7), it now suffices to show that $\lim _{\varepsilon \rightarrow 0} \mathbb{P}\left(\tau^{K} \neq \tau_{*}^{K}\right)=0$. Fix an arbitrary $T>0$ and $\kappa>0$. It then follows from Propositions 4.3, 4.4 and 4.5 that for each of the terms $\boldsymbol{\Psi}_{j}^{\varepsilon}$ appearing in (2.9), there exists a constant $C_{j}>0$ such that

$$
\mathbb{E} \sup _{t \in[0, T]} \boldsymbol{\Psi}_{j}^{\varepsilon}(t) \leq C_{j} \varepsilon^{\alpha_{j}-\kappa / 2},
$$

uniformly for all $\varepsilon \leq 1$. It then follows from Chebychev's inequality that

$$
\mathbb{P}\left(\tau^{K} \neq \tau_{*}^{K}\right) \leq \sum_{j} \mathbb{P}\left(\sup _{t \in[0, T]} \Psi_{j}^{\varepsilon}(t) \geq \varepsilon^{\alpha_{j}-\kappa}\right) \leq \sum_{j} C_{j} \varepsilon^{\kappa / 2},
$$

from which the claim follows.

Since (2.6) follows from (2.7) and (2.8), the proof of the theorem is complete if we show that (2.8) holds. Since it follows from Proposition 4.3 and Chebychev's inequality that

$$
\lim _{\varepsilon \rightarrow 0} \mathbb{P}\left(\sup _{t \leq T}\left\|\widetilde{\psi}_{\gamma}(t)-\psi_{\gamma}(t)\right\|_{L^{\infty}}>\varepsilon^{1 / 2-\kappa}\right)=0
$$

for every $T>0$, this claim follows at once.

\section{Analytic tools.}

3.1. Products and compositions of functions in Sobolev spaces. In this subsection, we collect some well-known bounds for products and compositions of functions in Sobolev spaces. As is usual in the analysis literature, we use the notation $\Phi \lesssim \Psi$ as a shorthand for "there exists a constant $C$ such that $\Phi \leq C \Psi$." These estimates will be useful in order control the various terms that arise in the Taylor expansion of the nonlinearity that will be performed in Section 5 below.

LeMma 3.1. Let $r, s, t \geq 0$ be such that $r \wedge s>t$ and $r+s>\frac{1}{2}+t$.

(1) For $f \in H^{r}$ and $g \in H^{s}$, we have $f g \in H^{t}$ and

$$
\|f g\|_{t} \lesssim\|f\|_{r}\|g\|_{s} .
$$


(2) For $f \in H^{r}$ and $g \in H^{-t}$, we have $f g \in H^{-s}$ and

$$
\|f g\|_{-s} \lesssim\|f\|_{r}\|g\|_{-t} .
$$

Proof. This result is very well known. A proof of (3.1) can be found, for example, in [10], Theorem 6.18, and (3.2) follows by duality.

Lemma 3.2. Let $s \in\left(\frac{1}{2}, 1\right)$. There exists $C>0$ such that for any $u \in H^{s}$ and any $G \in C^{1}\left(\mathbf{R}^{n} ; \mathbf{R}^{n}\right)$ satisfying

$$
\left\|G_{u}\right\|_{C^{1}}:=\sup \left\{|G(x)|+|\nabla G(x)|:|x| \leq\|u\|_{L^{\infty}}\right\}<\infty,
$$

we have

$$
\|G \circ u\|_{s} \leq C\left\|G_{u}\right\|_{C^{1}}\left(1+\|u\|_{s}\right) .
$$

Proof. Let $\tau_{h}$ be the shift operator defined by $\tau_{h} u(x):=u(x-h)$. It is well known (see, e.g., [8] or, for functions defined on $\mathbf{R}^{n}$, [1], Theorem 7.47) that the expression

$$
\|u\|_{L^{2}}+\left(\int_{0}^{1}\left[t^{-s} \sup _{|h|<t}\left\|u-\tau_{h} u\right\|_{L^{2}}\right]^{2} \frac{d t}{t}\right)^{1 / 2}
$$

defines an equivalent norm on $H^{s}$. The result then follows by inserting the estimates

$$
\begin{aligned}
\|G \circ u\|_{L^{2}} & \leq\|G \circ u\|_{L^{\infty}} \leq C\left\|G_{u}\right\|_{C^{1}}, \\
\left\|G \circ u-\tau_{h}(G \circ u)\right\|_{L^{2}} & \leq C\left\|G_{u}\right\|_{C^{1}}\left\|u-\tau_{h} u\right\|_{L^{2}}
\end{aligned}
$$

into (3.3).

3.2. Semigroup bounds. We will frequently use the fact that for $\alpha \geq \beta$ and $T>0$, there exists a constant $C>0$ such that

$$
\|S(t) u\|_{\alpha} \leq C t^{-(\alpha-\beta) / 2}\|u\|_{\beta}
$$

for any $\varepsilon \in(0,1], t \in[0, T]$ and $u \in H^{\beta}$. This is a straightforward consequence of standard analytic semigroup theory [10,14]. Since the generator of $S$ is selfadjoint in all of the $H^{s}$, it is also straightforward to prove (3.4) by hand. As a consequence, we have:

Lemma 3.3. Let $\alpha, \beta \in \mathbf{R}$ be such that $0 \leq \alpha-\beta<2$ and let $T>0$. There exists $C>0$ such that for all $t \in[0, T]$ and $u \in C\left([0, t] ; H^{\beta}\right)$ we have

$$
\left\|\int_{0}^{t} S(t-s) u(s) d s\right\|_{\alpha} \leq C t^{1-(\alpha-\beta) / 2} \sup _{s \in[0, t]}\|u(s)\|_{\beta} .
$$

Proof. It suffices to integrate the bound (3.4). 
The following bounds measure how well $S_{\varepsilon}$ approximates $S$ in these interpolation spaces. The general philosophy is that every power of $\varepsilon$ has to be paid with one spatial derivative worth of regularity. This type of powercounting is a direct consequence of the fact that the function $f$ that measures how much $\Delta_{\varepsilon}$ differs from $\partial_{x}^{2}$, is evaluated at $\varepsilon|k|$ in the definition of $\Delta_{\varepsilon}$. The precise bounds are the following:

Lemma 3.4. Let $\kappa \in[0,2]$. For $T>0$ there exists $C>0$ such that for any $t \in[0, T], \varepsilon \in(0,1]$, and $u \in H^{\beta}$, we have

$$
\begin{aligned}
\left\|S_{\varepsilon}(t) u-S(t) u\right\|_{\alpha} & \leq C \varepsilon^{\kappa} t^{-(\alpha-\beta+\kappa) / 2}\|u\|_{\beta} \quad(\beta \leq \alpha+2 \kappa), \\
\left\|S_{\varepsilon}(t) u\right\|_{\alpha} & \leq C t^{-(\alpha-\beta) / 2}\|u\|_{\beta} \quad(\beta \leq \alpha) .
\end{aligned}
$$

Proof. We set $\bar{f} \stackrel{\text { def }}{=} f-1$ and assume $\nu=1$ for notational simplicity, since the case $\nu \neq 1$ is virtually identical. The assumptions on $f$ imply that $|\bar{f}(\varepsilon n)| \leq c \varepsilon^{2} n^{2}$ whenever $n<\delta / \varepsilon$ and $\delta$ is some sufficiently small constant. Using the mean value theorem and the fact that we can assume $\delta<1$ without loss of generality, we obtain for $n<\delta / \varepsilon$ and $\kappa \in[0,2]$,

$$
\begin{aligned}
\left|\exp \left(-t n^{2} \bar{f}(\varepsilon n)\right)-1\right| & \leq\left(2 \wedge c t \varepsilon^{2} n^{4}\right) e^{c t \varepsilon^{2} n^{4}} \leq C t^{\kappa / 2} \varepsilon^{\kappa} n^{2 \kappa} e^{c t \delta^{2} n^{2}} \\
& \leq C \varepsilon^{\kappa} t^{\kappa / 2} n^{2 \kappa} e^{c \delta^{2} t\left(1+n^{2}\right)} .
\end{aligned}
$$

Inserting this bound into the identity

$$
\left(S_{\varepsilon}(t) u-S(t)\right) e_{n}=\left(e^{-t n^{2} \bar{f}(\varepsilon n)}-1\right) e^{-t\left(1+n^{2}\right)} e_{n},
$$

it then follows from (3.4) that

$$
\begin{aligned}
\left\|\Pi_{\delta / \varepsilon}\left(S_{\varepsilon}(t)-S(t)\right) u\right\|_{\alpha} & \leq C \varepsilon^{\kappa} t^{\kappa / 2}\left\|S\left(\left(1-\delta^{2} c\right) t\right) u\right\|_{\alpha+2 \kappa} \\
& \leq C \varepsilon^{\kappa} t^{-(\alpha-\beta+\kappa) / 2}\|u\|_{\beta},
\end{aligned}
$$

provided that we choose $\delta$ sufficiently small so that $\delta^{2} c \leq \frac{1}{2}$, say.

On the other hand, note that

$$
\left(I-\Pi_{\delta / \varepsilon}\right)\left(S_{\varepsilon}(t) u-S(t)\right) e_{n}=\mathbf{1}_{\{|n|>\delta / \varepsilon\}}\left(e^{-t n^{2} \bar{f}(\varepsilon n)}-1\right) e^{-t\left(1+n^{2}\right)} e_{n} .
$$

Recall that $\bar{f}(\varepsilon n) \geq q-1$ for all $n$, and that $q \in(0,1]$. Then we can find a constant $C$ such that

$$
\left|\exp \left(-t n^{2} \bar{f}(\varepsilon n)\right)-1\right| e^{-t\left(1+n^{2}\right)} \leq C e^{-q\left(1+n^{2}\right) t} .
$$

Moreover, for any $\kappa>0$ we have $\mathbf{1}_{\{|n|>\delta / \varepsilon\}} \leq|\varepsilon n / \delta|^{\kappa}$. It thus follows, using (3.4) again, that

$$
\left\|\left(I-\Pi_{\delta / \varepsilon}\right)\left(S_{\varepsilon}(t)-S(t)\right) u\right\|_{\alpha} \leq C \varepsilon^{\kappa}\|S(q t) u\|_{\alpha+\kappa} \leq C \varepsilon^{\kappa} t^{-(\alpha-\beta+\kappa) / 2}\|u\|_{\beta} .
$$

The bound (3.6) now follows by combining this inequality with (3.8). Inequality (3.7) follows by combining the special case $\kappa=0$ with (3.4). 
3.3. Estimates for the gradient term. In this section, we similarly show how well the operator $D_{\varepsilon}$ approximates $\partial_{x}$. Again, the guiding principle is that every power of $\varepsilon$ "costs" the loss of one derivative. However, we are also going to use the fact that $D_{\varepsilon}$ is a bounded operator. In this case, we can gain up to one spatial derivative with respect to the operator $\partial_{x}$, but we have to "pay" with the same number of inverse powers of $\varepsilon$. The rigorous statement for the latter fact is the following lemma.

Lemma 3.5. Let $\beta \in \mathbf{R}$ and $\alpha \in[0,1]$. There exists $C>0$ such that for all $\varepsilon \in(0,1]$ and $u \in H^{\beta}$ the estimate

$$
\left\|D_{\varepsilon} u\right\|_{\beta-\alpha} \leq C \varepsilon^{\alpha-1}\|u\|_{\beta}
$$

holds.

Proof. Using the assumption that $M:=|\mu|(\mathbf{R})<\infty$, together with Jensen's inequality and Fubini's theorem, we obtain

$$
\begin{aligned}
\left\|D_{\varepsilon} u\right\|_{L^{2}}^{2} & \leq \frac{1}{\varepsilon^{2}} \int\left(\int_{\mathbf{R}}|u(x+\varepsilon y)||\mu|(d y)\right)^{2} d x \\
& \leq \frac{M}{\varepsilon^{2}} \iint_{\mathbf{R}}|u(x+\varepsilon y)|^{2}|\mu|(d y) d x=\frac{M^{2}}{\varepsilon^{2}}\|u\|_{L^{2}}^{2} .
\end{aligned}
$$

On the other hand, assuming for the moment that $u$ is smooth, we use the assumption that $\mu(\mathbf{R})=0$, and apply Jensen's inequality and Minkowski's integral inequality to obtain

$$
\begin{aligned}
\left\|D_{\varepsilon} u\right\|_{L^{2}}^{2} & =\frac{1}{\varepsilon^{2}} \int\left(\int_{\mathbf{R}} u(x+\varepsilon y) \mu(d y)\right)^{2} d x \\
& =\frac{1}{\varepsilon^{2}} \int\left(\int_{\mathbf{R}} \int_{0}^{\varepsilon y} u^{\prime}(x+z) d z \mu(d y)\right)^{2} d x \\
& \leq \frac{M}{\varepsilon^{2}} \iint_{\mathbf{R}}\left(\int_{0}^{\varepsilon y}\left|u^{\prime}(x+z)\right| d z\right)^{2}|\mu|(d y) d x \\
& \leq \frac{M}{\varepsilon^{2}} \int_{\mathbf{R}}\left(\int_{0}^{\varepsilon y}\left(\int\left|u^{\prime}(x+z)\right|^{2} d x\right)^{1 / 2} d z\right)^{2}|\mu|(d y) \\
& =M\left\|u^{\prime}\right\|_{L^{2}}^{2} \int_{\mathbf{R}} y^{2}|\mu|(d y) \leq C\|u\|_{1}^{2} .
\end{aligned}
$$

Using complex interpolation, it follows that $\left\|D_{\varepsilon} u\right\|_{L^{2}} \leq C \varepsilon^{\alpha-1}\|u\|_{\alpha}$ for every $\alpha \in[0,1]$. The desired result then follows from the fact that $D_{\varepsilon}$ commutes with every Fourier multiplier.

The announced approximation result on the other hand is the following lemma. 
Lemma 3.6. Let $\beta \in \mathbf{R}$ and $\alpha \in[0,1]$. There exists $C>0$ such that for all $\varepsilon \in(0,1]$ and $u \in H^{\beta}$ the estimate

$$
\left\|D_{\varepsilon} u-\partial_{x} u\right\|_{\beta-1-\alpha} \leq C \varepsilon^{\alpha}\|u\|_{\beta}
$$

holds.

Proof. In view of (1.3) we have, assuming for the moment that $u$ is smooth,

$$
\left(D_{\varepsilon}-\partial_{x}\right) u(x)=\frac{1}{\varepsilon} \int_{\mathbf{R}} \int_{0}^{\varepsilon y} \int_{0}^{w} u^{\prime \prime}(x+z) d z d w \mu(d y) .
$$

Integrating against a test function $\varphi$ and applying Fubini's theorem, we arrive at

$$
\begin{aligned}
\left|\int \varphi(x)\left(D_{\varepsilon}-\partial_{x}\right) u(x) d x\right| & \leq \frac{C}{\varepsilon} \int_{\mathbf{R}} \int_{0}^{\varepsilon y} \int_{0}^{w}\|\varphi\|_{2-\beta}\|u\|_{\beta} d z d w|\mu|(d y) \\
& \leq C \varepsilon\|\varphi\|_{2-\beta}\|u\|_{\beta} \int_{\mathbf{R}}|y|^{2}|\mu|(d y),
\end{aligned}
$$

which implies that

$$
\left\|\left(D_{\varepsilon}-\partial_{x}\right) u\right\|_{\beta-2} \leq C \varepsilon\|u\|_{\beta} .
$$

On the other hand, Lemma 3.5 implies that

$$
\left\|\left(D_{\varepsilon}-\partial_{x}\right) u\right\|_{\beta-1} \leq C\|u\|_{\beta},
$$

and the result then follows as before by interpolating between these estimates.

As an immediate corollary of these bounds, we obtain the following useful fact.

Corollary 3.7. Let $\beta \in[0,1)$. There exists $C>0$ such that for $\varepsilon \in$ $(0,1], u \in H^{\beta}$, and $G \in C^{1}\left(\mathbf{R}^{n}\right)$ we have

$$
\left\|D_{\varepsilon} G(u)-\partial_{x} G(u)\right\|_{-1} \leq C \varepsilon^{\beta}\left\|G_{u}\right\|_{C^{1}}\left(1+\|u\|_{\beta}\right),
$$

where $\left\|G_{u}\right\|_{C^{1}}$ is defined as in Lemma 3.2.

Proof. Using Lemmas 3.6 and 3.2, we obtain

$$
\left\|D_{\varepsilon} G(u)-\partial_{x} G(u)\right\|_{-1} \leq C \varepsilon^{\beta}\|G(u)\|_{\beta} \leq C \varepsilon^{\beta}\left\|G_{u}\right\|_{C^{1}}\left(1+\|u\|_{\beta}\right),
$$

which is the stated claim.

4. Probabilistic tools. In this section, we prove some sharp estimates for certain expressions involving stochastic convolutions. Our main tool is the following version of Kolmogorov's continuity criterion, which follows 
immediately from the one given, for example, in [16]. The reason why we state condition (4.1) in this form, is that it is automatically satisfied (by hypercontractivity) for random fields taking values in a Wiener chaos of fixed (finite) order.

LEMma 4.1. Let $(\varphi(t))_{t \in[0,1]^{n}}$ be a Banach space-valued random field having the property that for any $q \in(2, \infty)$ there exists a constant $K_{q}>0$ such that

$$
\begin{gathered}
\left(\mathbb{E}\|\varphi(t)\|^{q}\right)^{1 / q} \leq K_{q}\left(\mathbb{E}\|\varphi(t)\|^{2}\right)^{1 / 2}, \\
\left(\mathbb{E}\|\varphi(s)-\varphi(t)\|^{q}\right)^{1 / q} \leq K_{q}\left(\mathbb{E}\|\varphi(s)-\varphi(t)\|^{2}\right)^{1 / 2}
\end{gathered}
$$

for all $s, t \in[0,1]^{n}$. Furthermore, suppose that the estimate

$$
\mathbb{E}\|\varphi(s)-\varphi(t)\|^{2} \leq K_{0}|s-t|^{\delta}
$$

holds for some $K_{0}, \delta>0$ and all $s, t \in[0,1]^{n}$. Then, for every $p>0$ there exists $C>0$ such that

$$
\mathbb{E} \sup _{t \in[0,1]^{n}}\|\varphi(t)\|^{p} \leq C\left(K_{0}+\mathbb{E}\|\varphi(0)\|^{2}\right)^{p / 2} .
$$

Throughout this subsection, we shall use $\theta_{k}$ and $\widetilde{\theta}_{k}$ for the Fourier coefficients of $\psi$ and $\widetilde{\psi}$, so that

$$
\psi(t)=\sum_{k \in \mathbf{Z}} \theta_{k}(t) e_{k}, \quad \widetilde{\psi}(t)=\sum_{k \in \mathbf{Z}} \widetilde{\theta}_{k}(t) e_{k} .
$$

With this notation at hand, we first state the following approximation bound, which shows that we can again trade powers of $k$ for powers of $\varepsilon$, provided that we look at the difference squared:

Lemma 4.2. For $t \geq 0, k \in \mathbf{Z}$ and $\varepsilon \in(0,1]$, we have

$$
\mathbb{E}\left|\widetilde{\theta}_{k}(t)-\theta_{k}(t)\right|^{2} \leq C\left(k^{-2} \wedge \varepsilon^{4} k^{2}\right) .
$$

Proof. We write again $\bar{f}=f-1$ and assume $\nu=1$ for simplicity. The Itô isometry then implies that

$$
\begin{aligned}
\mathbb{E}\left|\widetilde{\theta}_{k}(t)-\theta_{k}(t)\right|^{2}= & C \int_{0}^{\infty} e^{-2 t\left(1+k^{2}\right)}\left(1-h(\varepsilon|k|) e^{-t k^{2} \bar{f}(\varepsilon|k|)}\right)^{2} d t \\
\leq & C \int_{0}^{\infty} e^{-2 t\left(1+k^{2}\right)}\left(1-e^{-t k^{2} \bar{f}(\varepsilon|k|)}\right)^{2} d t \\
& +C \int_{0}^{\infty} e^{-2 t\left(1+k^{2}\right)} e^{-2 t k^{2} \bar{f}(\varepsilon|k|)}(1-h(\varepsilon|k|))^{2} d t \\
\stackrel{\text { def }}{=} & I_{1}+I_{2} .
\end{aligned}
$$


Let $\delta>0$ be a (small) constant to be determined later and consider first the term $I_{1}$ with $|\varepsilon k| \leq \delta$. Since $f$ is twice differentiable near the origin, we can find $\delta$ small enough so that $|\bar{f}(|\varepsilon k|)| \leq c \varepsilon^{2} k^{2}$ for some $c>0$. Therefore, for $t \geq 0$,

$$
\left|1-e^{-t k^{2} \bar{f}(\varepsilon|k|)}\right| \leq c t \varepsilon^{2} k^{4} e^{c t \varepsilon^{2} k^{4}} \leq c t \varepsilon^{2} k^{4} e^{c \delta^{2} t k^{2}},
$$

so that

$$
\left|I_{1}\right| \leq C \varepsilon^{4} k^{8} \int_{0}^{\infty} t^{2} e^{-2 t\left(1+k^{2}\right)+2 c \delta^{2} k^{2} t} d t
$$

If we ensure that $\delta$ is small enough so that $2 c \delta^{2} \leq 1$, we obtain

$$
\left|I_{1}\right| \leq C \varepsilon^{4} k^{8} \int_{0}^{\infty} t^{2} e^{-k^{2} t} d t \leq C \varepsilon^{4} k^{2} \leq C\left(k^{-2} \wedge \varepsilon^{4} k^{2}\right),
$$

where the last inequality follows from the fact that $|\varepsilon k| \leq \delta$ by assumption.

To treat the case $|\varepsilon k|>\delta$, we use the fact that by assumption there exists $q \in(0,1]$ such that $f \geq q$, so that

$$
\begin{aligned}
\left|I_{1}\right| & \leq \int_{0}^{\infty} e^{-2 t k^{2}}\left(1-e^{-t k^{2}(q-1)}\right)^{2} d t \leq C \int_{0}^{\infty} e^{-2 t q k^{2}} d t \\
& \leq C k^{-2} \leq C\left(k^{-2} \wedge \varepsilon^{4} k^{2}\right) .
\end{aligned}
$$

The bound on $I_{2}$ works in pretty much the same way, using the fact that the assumptions on $h$ imply that

$$
|1-h(\varepsilon|k|)| \leq C\left(1 \wedge \varepsilon^{2} k^{2}\right) .
$$

Using again the fact that $f \geq q$, we then obtain

$$
I_{2} \leq C \int_{0}^{\infty} e^{-2 t q k^{2}}\left(1 \wedge \varepsilon^{4} k^{4}\right) d t \leq C\left(k^{-2} \wedge \varepsilon^{4} k^{2}\right)
$$

as required.

We continue with a sequence of propositions, in which the estimates obtained in the previous lemma are used to establish various bounds for stochastic convolutions.

Proposition 4.3. Let $0<\gamma<\chi$. For $\kappa>0$ and $\varepsilon \in(0,1]$ we have

$$
\begin{array}{ll}
\mathbb{E} \sup _{t \in[0, T]}\left\|\psi_{\gamma}(t)\right\|_{L^{\infty}} \leq C \varepsilon^{\gamma / 2-\kappa}, & \mathbb{E} \sup _{t \in[0, T]}\left\|\widetilde{\psi}_{\gamma}(t)\right\|_{L^{\infty}} \leq C \varepsilon^{\gamma / 2-\kappa}, \\
\mathbb{E} \sup _{t \in[0, T]}\left\|\widetilde{\psi}_{\gamma}^{\chi}(t)\right\|_{L^{\infty}} \leq C \varepsilon^{\gamma / 2-\kappa}, & \mathbb{E} \sup _{t \in[0, T]}\left\|\widetilde{\psi}_{\gamma}(t)-\psi_{\gamma}(t)\right\|_{L^{\infty}} \leq C \varepsilon^{1 / 2-\kappa} .
\end{array}
$$


Proof. We start with the proof of the second estimate. Observe that $\widetilde{\theta}_{k}$ is a complex one-dimensional stationary Ornstein-Uhlenbeck process with variance $h^{2} /\left(2\left(1+\nu k^{2} f\right)\right)$ and characteristic time $1+\nu k^{2} f$. This implies that

$$
\mathbb{E}\left|\widetilde{\theta}_{k}(t)\right|^{2}=\frac{h^{2}(\varepsilon|k|)}{2\left(1+\nu k^{2} f(\varepsilon|k|)\right)} \leq C\left(1 \wedge k^{-2}\right)
$$

and

$$
\mathbb{E}\left|\widetilde{\theta}_{k}(t)-\widetilde{\theta}_{k}(s)\right|^{2} \leq C h^{2}(\varepsilon|k|)|t-s| \leq C|t-s| .
$$

These bounds imply that, on the one hand,

$$
\mathbb{E}\left|\widetilde{\theta}_{k}(t) e_{k}(x)-\widetilde{\theta}_{k}(s) e_{k}(y)\right|^{2} \leq C \mathbb{E}\left|\widetilde{\theta}_{k}(t)\right|^{2}+C \mathbb{E}\left|\widetilde{\theta}_{k}(s)\right|^{2} \leq C\left(1 \wedge k^{-2}\right),
$$

while on the other hand, one has

$$
\begin{aligned}
& \mathbb{E}\left|\widetilde{\theta}_{k}(t) e_{k}(x)-\widetilde{\theta}_{k}(s) e_{k}(y)\right|^{2} \\
& \quad \leq C \mathbb{E}\left|\widetilde{\theta}_{k}(t)-\widetilde{\theta}_{k}(s)\right|^{2}+C k^{2}|x-y|^{2} \mathbb{E}\left|\widetilde{\theta}_{k}(s)\right|^{2} \\
& \quad \leq C|t-s|+C|x-y|^{2} .
\end{aligned}
$$

Combining these inequalities we find that, for every $\kappa \in[0,2]$,

$$
\mathbb{E}\left|\widetilde{\theta}_{k}(t) e_{k}(x)-\widetilde{\theta}_{k}(s) e_{k}(y)\right|^{2} \leq C\left(1 \wedge k^{-2}\right)^{1-\kappa / 2}\left(|t-s|+|x-y|^{2}\right)^{\kappa / 2} .
$$

Since the $\widetilde{\theta}_{k}$ 's are independent except for the reality condition $\widetilde{\theta}_{-k}=\overline{\widetilde{\theta}}_{k}$, we infer that

$$
\begin{aligned}
\mathbb{E}\left|\widetilde{\psi}_{\gamma}(t, x)-\widetilde{\psi}_{\gamma}(s, y)\right|^{2} & \leq C \sum_{|k|>\varepsilon^{-\gamma}} \mathbb{E}\left|\widetilde{\theta}_{k}(t) e_{k}(x)-\widetilde{\theta}_{k}(s) e_{k}(y)\right|^{2} \\
& \leq C\left(|t-s|+|x-y|^{2}\right)^{\kappa / 2} \sum_{|k|>\varepsilon^{-\gamma}}\left(1 \wedge k^{-2}\right)^{1-\kappa / 2} \\
& \leq C \varepsilon^{(1-\kappa) \gamma}\left(|t-s|+|x-y|^{2}\right)^{\kappa / 2}
\end{aligned}
$$

Arguing similarly, we obtain

$$
\mathbb{E}\left|\widetilde{\psi}_{\gamma}(0,0)\right|^{2} \leq C \sum_{|k|>\varepsilon^{-\gamma}} \mathbb{E}\left|\tilde{\theta}_{k}(0)\right|^{2} \leq C \sum_{|k|>\varepsilon^{-\gamma}}\left(1 \wedge k^{-2}\right) \leq C \varepsilon^{\gamma} .
$$

The result now follows by combining these two bounds with Lemma 4.1.

The proof of the first and third estimates being very similar, we do not reproduce them here. In order to prove the last estimate, we use Lemma 4.2 to obtain

$$
\mathbb{E}\left|\widetilde{\theta}_{k}(t)-\theta_{k}(t)\right|^{2} \leq C\left(k^{-2}\right)^{3 / 4+\kappa / 4}\left(\varepsilon^{4} k^{2}\right)^{1 / 4-\kappa / 4} \leq C \varepsilon^{1-\kappa} k^{-1-\kappa} .
$$

This bound then replaces (4.6), and the rest of the proof is again analogous to the proof of the second estimate. 
Proposition 4.4. Let $\zeta>0$. For $\kappa>0$ and $\varepsilon \in(0,1]$, we have

$$
\begin{aligned}
\mathbb{E} \sup _{t \in[0, T]}\left\|\psi^{\zeta}(t)\right\|_{\alpha} \leq C \varepsilon^{-\zeta(\alpha-1 / 2)-\kappa} & \left(\alpha>\frac{1}{2}\right), \\
\mathbb{E} \sup _{t \in[0, T]}\left\|\widetilde{\psi}^{\zeta}(t)-\psi^{\zeta}(t)\right\|_{\alpha} \leq C \varepsilon^{2-\zeta(\alpha+3 / 2)-\kappa} & \left(\alpha>-\frac{3}{2}\right) .
\end{aligned}
$$

Proof. In view of the estimates

$$
\mathbb{E}\left|\theta_{k}(t)\right|^{2} \leq C k^{-2}, \quad \mathbb{E}\left|\theta_{k}(t)-\theta_{k}(s)\right|^{2} \leq C|t-s|,
$$

we obtain

$$
\begin{aligned}
\mathbb{E}\left\|\psi^{\zeta}(t)-\psi^{\zeta}(s)\right\|_{\alpha}^{2} & \leq C|t-s|^{\kappa} \sum_{|k| \leq \varepsilon^{-\zeta}}\left(1+k^{2}\right)^{\alpha-1+\kappa} \\
& \leq C|t-s|^{\kappa} \varepsilon^{-2 \zeta(\alpha-1 / 2+\kappa)}
\end{aligned}
$$

and

$$
\mathbb{E}\left\|\psi^{\zeta}(0)\right\|_{\alpha}^{2} \leq C \varepsilon^{-2 \zeta(\alpha-1 / 2)} .
$$

Inequality (4.8) thus follows from Lemma 4.1.

In order to prove (4.9), we argue similarly, but the estimates are slightly more involved. Write $\delta_{k}:=\widetilde{\theta}_{k}-\theta_{k}$ so that $\widetilde{\psi}^{\zeta}-\psi^{\zeta}=\sum_{|k| \leq \varepsilon^{-\zeta}} \delta_{k} e_{k}$. Using (4.7) and (4.10), we have for $s, t \geq 0$,

$$
\mathbb{E}\left|\delta_{k}(t)-\delta_{k}(s)\right|^{2} \leq C|t-s| .
$$

Combining this bound with Lemma 4.2, we infer that for $\kappa \in\left[0, \frac{1}{2}\right)$,

$$
\mathbb{E}\left|\delta_{k}(t)-\delta_{k}(s)\right|^{2} \leq C\left(k^{-2}\right)^{\kappa}\left(\varepsilon^{4} k^{2}\right)^{1-2 \kappa}|t-s|^{\kappa}=C \varepsilon^{4-8 \kappa} k^{2-6 \kappa}|t-s|^{\kappa} .
$$

For $\kappa \in\left(0, \frac{1}{3} \alpha+\frac{1}{2}\right)$, we thus obtain

$$
\begin{aligned}
\mathbb{E}\left\|\left(\widetilde{\psi}^{\zeta}-\psi^{\zeta}\right)(t)-\left(\widetilde{\psi}^{\zeta}-\psi^{\zeta}\right)(s)\right\|_{\alpha}^{2} & \leq C|t-s|^{\kappa} \varepsilon^{4-8 \kappa} \sum_{|k| \leq \varepsilon^{-\zeta}}\left(1+k^{2}\right)^{\alpha+1-3 \kappa} \\
& \leq C|t-s|^{\kappa} \varepsilon^{4-\zeta(2 \alpha+3)-8 \kappa}
\end{aligned}
$$

and similarly

$$
\mathbb{E} \sup _{t \in[0, T]}\left\|\widetilde{\psi}^{\zeta}(t)-\psi^{\zeta}(t)\right\|_{\alpha}^{2} \leq C \varepsilon^{4-\zeta(2 \alpha+3)-8 \kappa} .
$$

The desired estimate (4.9) now follows from Lemma 4.1.

Proposition 4.5. Let $\zeta>0$. For every $\kappa>0$ there exists $C>0$ such that

$$
\mathbb{E} \sup _{t \in[0, T]} \Theta\left(\widetilde{\psi}_{\zeta}(t)\right) \leq C \varepsilon^{-1+(\zeta-1)^{+}-\kappa}
$$

for all $\varepsilon \in(0,1]$, where we wrote $(\zeta-1)^{+} \stackrel{\text { def }}{=} 0 \vee(\zeta-1)$. 
Proof. As in the proof of Propositions 4.3 and 4.4, we shall apply Kolmogorov's continuity criterion from Lemma 4.1, this time for $L^{2}$-valued random fields. It follows from (4.6) that

$$
\begin{aligned}
\mathbb{E}\left\|\widehat{D}_{\varepsilon y}\left(\widetilde{\psi}_{\zeta}(t)-\widetilde{\psi}_{\zeta}(s)\right)\right\|_{L^{2}}^{2} & =\sum_{|k|>\varepsilon^{-\zeta}}\left|\frac{e^{i k \varepsilon y}-1}{\varepsilon y}\right|^{2} \mathbb{E}\left|\widetilde{\theta}_{k}(t)-\widetilde{\theta}_{k}(s)\right|^{2} \\
& \leq C \sum_{k>\varepsilon^{-\zeta}} \frac{1-\cos (k \varepsilon y)}{|\varepsilon k y|^{2}} .
\end{aligned}
$$

Note that, up to a factor $\varepsilon|y|$, this sum can be interpreted as a Riemann sum for the function $H(t) \stackrel{\text { def }}{=} t^{-2}(1-\cos (t))$. In fact, since $H(t) \leq 2\left(1 \wedge t^{-2}\right)$,

$$
\begin{aligned}
\varepsilon|y| \sum_{k>\varepsilon^{-\zeta}} \frac{1-\cos (k \varepsilon y)}{|k \varepsilon y|^{2}} & =\sum_{k>\varepsilon^{-\zeta}} \varepsilon|y| H(k \varepsilon y) \leq 2 \int_{\varepsilon^{1-\zeta}}^{\infty}\left(1 \wedge t^{-2}\right) d t \\
& \leq C \varepsilon^{(\zeta-1)^{+}} .
\end{aligned}
$$

It thus follows that

$$
\mathbb{E}\left\|\widehat{D}_{\varepsilon y}\left(\widetilde{\psi}_{\zeta}(t)-\widetilde{\psi}_{\zeta}(s)\right)\right\|_{L^{2}}^{2} \leq C|\varepsilon y|^{-1} \varepsilon^{(\zeta-1)^{+}} .
$$

On the other hand, (4.6) and (4.7) imply that

$$
\mathbb{E}\left|\widetilde{\theta}_{k}(t)-\widetilde{\theta}_{k}(s)\right|^{2} \leq C\left(1 \wedge k^{-2}\right)^{3 / 4}|t-s|^{1 / 4},
$$

and therefore

$$
\begin{aligned}
\mathbb{E}\left\|\widehat{D}_{\varepsilon y}\left(\widetilde{\psi}_{\zeta}(t)-\widetilde{\psi}_{\zeta}(s)\right)\right\|_{L^{2}}^{2} & =\sum_{|k|>\varepsilon^{-\zeta}}\left|\frac{e^{i k \varepsilon y}-1}{\varepsilon y}\right|^{2} \mathbb{E}\left|\widetilde{\theta}_{k}(t)-\widetilde{\theta}_{k}(s)\right|^{2} \\
& \leq C|\varepsilon y|^{-2}|t-s|^{1 / 4} \sum_{|k|>\varepsilon^{-\zeta}}\left(1 \wedge k^{-2}\right)^{3 / 4} \\
& \leq C|\varepsilon y|^{-2}|t-s|^{1 / 4}
\end{aligned}
$$

Combining (4.12) and (4.13), we find that

$$
\mathbb{E}\left\|\widehat{D}_{\varepsilon y}\left(\widetilde{\psi}_{\zeta}(t)-\widetilde{\psi}_{\zeta}(s)\right)\right\|_{L^{2}}^{2} \leq|\varepsilon y|^{-1-\kappa}|t-s|^{\kappa / 4} \varepsilon^{(1-\kappa)(\zeta-1)^{+}} .
$$

Similarly, we obtain

$$
\begin{aligned}
\mathbb{E}\left\|\widehat{D}_{\varepsilon y} \widetilde{\psi}_{\zeta}(0)\right\|_{L^{2}}^{2} & =\sum_{|k|>\varepsilon^{-\zeta}}\left|\frac{e^{i k \varepsilon y}-1}{\varepsilon y}\right|^{2} \mathbb{E}\left|\widetilde{\theta}_{k}(0)\right|^{2} \\
& \leq C \sum_{|k|>\varepsilon^{-\zeta}} \frac{1-\cos (k \varepsilon y)}{|\varepsilon k y|^{2}} \leq C|\varepsilon y|^{-1} \varepsilon^{(\zeta-1)^{+}} .
\end{aligned}
$$


In view of Lemma 4.1, the latter two estimates imply that

$$
\mathbb{E} \sup _{t \in[0, T]}\left\|\widehat{D}_{\varepsilon y} \widetilde{\psi}_{\zeta}(t)\right\|_{L^{2}}^{2} \leq C|\varepsilon y|^{-1-\kappa} \varepsilon^{(1-\kappa)(\zeta-1)^{+}} .
$$

Using this bound, the desired result for $\Theta\left(\widetilde{\psi}_{\zeta}(t)\right)$ can be obtained easily, since

$$
\begin{aligned}
\mathbb{E} \sup _{t \in[0, T]} \Theta\left(\widetilde{\psi}_{\zeta}(t)\right) & =\mathbb{E} \sup _{t \in[0, T]} \int_{\mathbf{R}}|y|^{2}\left\|\widehat{D}_{\varepsilon y} \widetilde{\psi}_{\zeta}(t)\right\|_{L^{2}}^{2}|\mu|(d y) \\
& \leq \int_{\mathbf{R}}|y|^{2} \mathbb{E} \sup _{t \in[0, T]}\left\|\widehat{D}_{\varepsilon y} \widetilde{\psi}_{\zeta}(t)\right\|_{L^{2}}^{2}|\mu|(d y) \\
& \leq C \varepsilon^{-1-\kappa+(1-\kappa)(\zeta-1)^{+}} \int_{\mathbf{R}}|y|^{1-\kappa}|\mu|(d y) \\
& \leq C \varepsilon^{-1-\kappa+(1-\kappa)(\zeta-1)^{+}} .
\end{aligned}
$$

The result now follows by rescaling $\kappa$.

The next and final result of this section involves the term which gives rise to the correction term in the limiting equation. Before stating the result, we introduce the notation

$$
\begin{aligned}
\Xi_{\varepsilon}^{y}(u) & \stackrel{\text { def }}{=} \frac{\varepsilon y^{2}}{2} \widehat{D}_{\varepsilon y} u \otimes \widehat{D}_{\varepsilon y} u, \\
\Lambda^{y} & \stackrel{\text { def }}{=} \frac{1}{2 \pi \nu} \int_{\mathbf{R}_{+}} \frac{h^{2}(t)}{t^{2} f(t)}(1-\cos (y t)) d t
\end{aligned}
$$

and

$$
\Lambda_{\varepsilon}^{y} \stackrel{\text { def }}{=} \sum_{\varepsilon^{-\gamma}<k<\varepsilon^{-\chi}} \Lambda_{\varepsilon, k}^{y} \stackrel{\text { def }}{=} \sum_{\varepsilon^{-\gamma}<k<\varepsilon^{-\chi}} \frac{(1-\cos (\varepsilon k y)) h^{2}(\varepsilon k)}{2 \pi \varepsilon\left(1+\nu k^{2} f(\varepsilon k)\right)} .
$$

Note that one has the identities

$$
\Xi_{\varepsilon}(u)=\int_{\mathbf{R}} \Xi_{\varepsilon}^{y}(u) \mu(d y), \quad \Lambda=\int_{\mathbf{R}} \Lambda^{y} \mu(d y), \quad \mathbb{E} \Xi_{\varepsilon}^{y}\left(\widetilde{\psi}_{\gamma}^{\chi}\right)=\Lambda_{\varepsilon}^{y} I,
$$

where the constant $\Lambda$ is given by (1.5).

Proposition 4.6. Let $\alpha>\frac{1}{2}, \gamma \leq \frac{1}{2}$ and $\chi \geq \frac{3}{2}$. For $\varepsilon \in(0,1]$, we then have

$$
\mathbb{E} \sup _{t \in[0, T]}\left\|\Lambda-\Xi_{\varepsilon}\left(\widetilde{\psi}_{\gamma}^{\chi}(t)\right)\right\|_{-\alpha} \leq C \varepsilon^{1 / 2}
$$

Proof. The proof is an application of Lemma 4.1 with $\xi=\Lambda-\Xi_{\varepsilon}\left(\widetilde{\psi}_{\gamma}^{\chi}\right)$. For brevity, we shall write $A:=\Xi_{\varepsilon}\left(\widetilde{\psi}_{\gamma}^{\chi}\right)$ and $A^{y}:=\Xi_{\varepsilon}^{y}\left(\widetilde{\psi}_{\gamma}^{\chi}\right)$. We divide the proof into several steps. 
Step 1. First, we claim that $\xi(t)=\Lambda-A(t)$ satisfies the condition (4.1) concerning the equivalence of all $q$-moments.

To see this, note that $\widetilde{\psi}_{\gamma}^{\chi}$ admits the representation $\widetilde{\psi}(t)=\sum_{k} \alpha_{k}(t) e_{k}$ where each $\alpha_{k}(t)$ is a Gaussian random vector in $\mathbf{R}^{n}$. As a consequence, for every $y \in \mathbf{R}$, each component of $\Lambda_{\varepsilon}^{y}-A^{y}$ is a polynomial of Gaussian random variables of degree at most two. It thus belongs to the direct sum of Wiener chaoses of order $\leq 2$ and the same is true for $\Lambda_{\varepsilon}-A$, since each Wiener chaos is a closed subspace of the space of square integrable random variables. The claim thus follows from the well-known equivalence of moments for Hilbert space-valued Wiener chaos (see, e.g., [13]).

Step 2. In this step, we estimate how well $\Lambda_{\varepsilon}^{y}$ approximates $\Lambda^{y}$. Since $|1-\cos x| \leq C\left(1 \wedge x^{2}\right)$, we have the bound $\left|\Lambda_{\varepsilon, k}^{y}\right| \leq C\left(\varepsilon y^{2} \wedge\left(\varepsilon k^{2}\right)^{-1}\right)$ for some constant $C$. As an immediate consequence, we have the bound

$$
\left|\Lambda_{\varepsilon}^{y}-\sum_{k \geq 1} \Lambda_{\varepsilon, k}^{y}\right| \leq C\left(\varepsilon^{1-\gamma} y^{2}+\varepsilon^{\chi-1}\right) .
$$

Define now the function

$$
\Phi_{y}(t)=\frac{(1-\cos (y t)) h^{2}(t)}{2 \pi \nu t^{2} f(t)}
$$

so that, since $h^{2} / f$ is bounded by assumption, we obtain the bound

$$
\left|\Lambda_{\varepsilon, k}^{y}-\varepsilon \Phi_{y}(\varepsilon k)\right| \leq C \frac{\varepsilon y^{2}}{k^{2}} .
$$

Combining this bound with (4.14), we have

$$
\left|\Lambda_{\varepsilon}^{y}-\sum_{k \geq 1} \varepsilon \Phi_{y}(\varepsilon k)\right| \leq C\left(\varepsilon^{1-\gamma} y^{2}+\varepsilon^{\chi-1}\right) .
$$

At this stage, we recall that for any function $\Phi$ of bounded variation, one has the approximation

$$
\left|\sum_{k \geq 1} \varepsilon \Phi(\varepsilon k)-\int_{0}^{\infty} \Phi(t) d t\right| \leq \varepsilon\|\Phi\|_{\mathrm{BV}},
$$

where $\|\Phi\|_{\mathrm{BV}}$ denotes the variation of $\Phi$ over $\mathbf{R}_{+}$. Furthermore, for any pair $\Phi, \Psi$, we have the bound

$$
\|\Phi \Psi\|_{\mathrm{BV}} \leq\|\Phi\|_{L^{\infty}}\|\Psi\|_{\mathrm{BV}}+\|\Psi\|_{L^{\infty}}\|\Phi\|_{\mathrm{BV}} .
$$

If we set $\Psi_{y}(t)=(1-\cos (y t)) / t^{2}$, we have

$$
\begin{aligned}
\left\|\Psi_{y}\right\|_{\mathrm{BV}} & =\int_{0}^{\infty}\left|\Psi_{y}^{\prime}(t)\right| d t=\int_{0}^{\infty} \frac{|y t \sin y t+2 \cos y t-2|}{t^{3}} d t \\
& \leq C|y|^{3} \int_{0}^{\infty}\left(1 \wedge \frac{1}{y^{2} t^{2}}\right) d t \leq C y^{2} .
\end{aligned}
$$


Since $\Psi(0)=y^{2} / 2$, a similar bound holds for its $L^{\infty}$ norm, and we conclude from (4.15) that

$$
\left\|\Phi_{y}\right\|_{\mathrm{BV}} \leq C y^{2} \text {. }
$$

It follows immediately that we have the bound

$$
\left|\Lambda_{\varepsilon}^{y}-\Lambda^{y}\right| \leq C\left(\varepsilon^{1-\gamma} y^{2}+\varepsilon y^{2}+\varepsilon^{\chi-1}\right)
$$

Step 3. We now use these bounds in order to obtain control over $\|\Lambda-A\|_{-\alpha}^{2}$ for a fixed time $t \geq 0$ (which is often suppressed in the notation).

In order to shorten the notation, note that, we can write

$$
\widetilde{\psi}(x, t)=\sum_{k \in \mathbf{Z}} \frac{h(\varepsilon|k|)}{\sqrt{2} \sqrt{1+\nu k^{2} f(\varepsilon|k|)}} \eta_{k}(t) e_{k}(x),
$$

where the $\eta_{k}$ are a sequence of i.i.d. $\mathbf{C}^{n}$-valued Ornstein-Uhlenbeck processes with

$$
\mathbb{E}\left(\eta_{k}(t) \otimes \eta_{\ell}(s)\right)=\mathcal{E}_{k}^{t-s} \delta_{k,-\ell} I, \quad \mathcal{E}_{k}^{t}=\exp \left(-\left(1+\nu k^{2} f(\varepsilon|k|)\right)|t|\right),
$$

and satisfying the reality condition $\eta_{-k}=\bar{\eta}_{k}$. Here, $I$ denotes the identity matrix. We will also use the notational shortcut

$$
\mathbf{A}_{k, \ell}^{t} \stackrel{\text { def }}{=} \eta_{k}(t) \otimes \eta_{\ell}(t) \text {. }
$$

Set now

$$
q_{\varepsilon}^{k}=\frac{e^{i k \varepsilon y}-1}{\sqrt{2}} \frac{h(\varepsilon|k|)}{\sqrt{1+\nu k^{2} f(\varepsilon|k|)}},
$$

as a shorthand. With all of this notation in place, it follows from the definition of $\Lambda_{\varepsilon}^{y}$ that

$$
A^{y}(t)-\Lambda_{\varepsilon}^{y} I=\sum_{\varepsilon^{-\gamma}<|k|,|\ell| \leq \varepsilon^{-\chi}} q_{\varepsilon}^{k} q_{\varepsilon}^{\ell}\left(\mathbf{A}_{k, \ell}^{t}-\delta_{k,-\ell} I\right) e_{k+\ell} .
$$

As a consequence, we have the identity

$$
\begin{aligned}
& \mathbb{E}\left\|\Lambda_{\varepsilon}^{y} I-A^{y}(t)\right\|_{-\alpha}^{2} \\
& =\sum_{k \in \mathbf{Z}}\left(1+|k|^{2}\right)^{-\alpha} \sum_{\ell, m} q_{\varepsilon}^{\ell} q_{\varepsilon}^{k-\ell} \bar{q}_{\varepsilon}^{m} \bar{q}_{\varepsilon}^{k-m} \\
& \quad \times \mathbb{E} \operatorname{tr}\left(\left(\mathbf{A}_{\ell, k-\ell}^{t}-\delta_{k, 0} I\right)\left(\overline{\mathbf{A}}_{m, k-m}^{t}-\delta_{k, 0} I\right)\right),
\end{aligned}
$$

where the second sum ranges over all $\ell, m \in \mathbf{Z}$ for which $\ell, k-\ell, m, k-m$ belong to $\left(\varepsilon^{-\gamma}, \varepsilon^{-\chi}\right]$. A straightforward case analysis allows to check that

$$
\mathbb{E} \operatorname{tr}\left(\left(\mathbf{A}_{\ell, k-\ell}^{t}-\delta_{k, 0} I\right)\left(\overline{\mathbf{A}}_{m, k-m}^{t}-\delta_{k, 0} I\right)\right)=n \delta_{\ell, m}+n^{2} \delta_{\ell, k-m},
$$

so that

$$
\mathbb{E}\left\|\Lambda_{\varepsilon}^{y} I-A^{y}(t)\right\|_{-\alpha}^{2} \leq C \sum_{k \in \mathbf{Z}}\left(1+|k|^{2}\right)^{-\alpha} \sum_{\ell \in \mathbf{Z}}\left|q_{\varepsilon}^{\ell}\right|^{2}\left|q_{\varepsilon}^{k-\ell}\right|^{2} .
$$


Note now that there exists a constant $C$ such that the bound

$$
\left|q_{\varepsilon}^{k}\right| \leq C \sqrt{\varepsilon}\left(|y| \wedge \frac{1}{\varepsilon|k|}\right) \leq C \varepsilon^{(1-\beta) / 2}|k|^{-\beta / 2}|y|^{1-\beta / 2}
$$

is valid for all $\varepsilon<1, k \in \mathbf{Z}, y \in \mathbf{R}$, and $\beta \in[0,1]$. It follows that there exists a constant $C>0$ such that we have the bound

$$
\begin{aligned}
\mathbb{E}\left\|\Lambda_{\varepsilon}^{y} I-A^{y}(t)\right\|_{-\alpha}^{2} & \leq C \sum_{\ell, m \geq 1} \frac{\left|q_{\varepsilon}^{\ell}\right|^{2}\left|q_{\varepsilon}^{m}\right|^{2}}{|\ell+m|^{2 \alpha}} \leq C \sum_{\ell, m \geq 1} \frac{\left|q_{\varepsilon}^{\ell}\right|^{2}\left|q_{\varepsilon}^{m}\right|^{2}}{|\ell|^{\alpha}|m|^{\alpha}} \\
& \leq C \varepsilon^{2-2 \beta} \sum_{\ell, m \geq 1} \frac{|y|^{4-2 \beta}}{|\ell|^{\alpha+\beta}|m|^{\alpha+\beta}} \leq C \varepsilon|y|^{3},
\end{aligned}
$$

where we made the choice $\beta=\frac{1}{2}$ to obtain the last bound, using the fact that $\alpha>\frac{1}{2}$ by assumption. Combining this bound with (4.16), the constraints $\gamma \leq \frac{1}{2}$ and $\chi \geq \frac{3}{2}$, and using the fact that $\mu$ has finite fourth moment, we have

$$
\begin{aligned}
\mathbb{E}\|\Lambda I-A(t)\|_{-\alpha}^{2} & \leq C \int \mathbb{E}\left\|\Lambda^{y}-A^{y}(t)\right\|_{-\alpha}^{2}|\mu|(d y) \\
& \leq C \int \mathbb{E}\left\|\Lambda_{\varepsilon}^{y}-A^{y}(t)\right\|_{-\alpha}^{2}|\mu|(d y)+C \varepsilon \\
& \leq C \varepsilon .
\end{aligned}
$$

Step 4. Finally, we shall estimate $\mathbb{E}\|A(t)-A(s)\|_{-\alpha}^{2}$. Similarly to (4.17), this involves the identity

$$
\mathbb{E} \operatorname{tr}\left(\mathbf{A}_{\ell, k-\ell}^{t} \overline{\mathbf{A}}_{m, k-m}^{s}\right)=n \delta_{k, 0}+\left(n \delta_{l, m}+n^{2} \delta_{l, k-m}\right) \mathcal{E}_{\ell}^{t-s} \mathcal{E}_{k-m}^{t-s} .
$$

As a consequence, we infer that

$$
\begin{aligned}
D_{k \ell m}(t, s) & \stackrel{\text { def }}{=} \mathbb{E} \operatorname{tr}\left(\left(\mathbf{A}_{\ell, k-\ell}^{t}-\mathbf{A}_{\ell, k-\ell}^{s}\right)\left(\overline{\mathbf{A}}_{m, k-m}^{t}-\overline{\mathbf{A}}_{m, k-m}^{s}\right)\right) \\
& =2\left(n \delta_{\ell, m}+n^{2} \delta_{\ell, k-m}\right)\left(1-\mathcal{E}_{\ell}^{t-s} \mathcal{E}_{k-m}^{t-s}\right) .
\end{aligned}
$$

It thus follows that for any $\delta \in[0,1]$,

$$
\begin{aligned}
& D_{k \ell m}(t, s) \\
& \quad \leq C\left(\delta_{\ell, m}+\delta_{\ell, k-m}\right)\left(1 \wedge\left(2+\nu \ell^{2} f(\varepsilon|\ell|)+\nu(k-m)^{2} f(\varepsilon|k-m|)\right)|t-s|\right) \\
& \quad \leq C\left(\delta_{\ell, m}+\delta_{\ell, k-m}\right)|t-s|^{\delta}\left(1+\ell^{2 \delta} f(\varepsilon|\ell|)^{\delta}+(k-m)^{2 \delta} f(\varepsilon|k-m|)^{\delta}\right) .
\end{aligned}
$$

Using this bound, we obtain

$$
\begin{aligned}
& \mathbb{E}\left\|A^{y}(t)-A^{y}(s)\right\|_{-\alpha}^{2} \\
& =\sum_{k \in \mathbf{Z}}\left(1+|k|^{2}\right)^{-\alpha} \sum_{\ell, m} q_{\varepsilon}^{\ell} q_{\varepsilon}^{k-\ell} \bar{q}_{\varepsilon}^{m} \bar{q}_{\varepsilon}^{k-m} D_{k \ell m}(t, s)
\end{aligned}
$$




$$
\begin{aligned}
& \leq \sum_{k \in \mathbf{Z}}\left(1+|k|^{2}\right)^{-\alpha} \sum_{\ell}\left|q_{\varepsilon}^{\ell}\right|^{2}\left|q_{\varepsilon}^{k-\ell}\right|^{2}\left(D_{k \ell \ell}(t, s)+D_{k, \ell, k-\ell}(t, s)\right) \\
& \leq C|t-s|^{\delta} \sum_{k \in \mathbf{Z}}\left(1+|k|^{2}\right)^{-\alpha} \sum_{\ell}\left|q_{\varepsilon}^{\ell}\right|^{2}\left|q_{\varepsilon}^{k-\ell}\right|^{2} \\
& \quad \times\left(1+\ell^{2 \delta} f(\varepsilon|\ell|)^{\delta}+|k-\ell|^{2 \delta} f(\varepsilon|k-\ell|)^{\delta}\right) .
\end{aligned}
$$

Note that this expression is almost the same as in Step 3. Using the calculations done there and taking into account that $h$ and $h / f^{2}$ are bounded functions, we infer that

$$
\mathbb{E}\left\|A^{y}(t)-A^{y}(s)\right\|_{-\alpha}^{2}=C|t-s|^{\delta} \sum_{\ell, m \geq 1} \frac{\left|q_{\varepsilon}^{\ell}\right|^{2}\left|q_{\varepsilon}^{m}\right|^{2}}{|\ell|^{\alpha-2 \delta}|m|^{\alpha-2 \delta}} \leq C|t-s|^{\delta} \varepsilon|y|^{3},
$$

and therefore, using Jensen's inequality (which can be applied since $|\mu|$ has finite mass), and Fubini's theorem,

$$
\begin{aligned}
\mathbb{E}\|A(t)-A(s)\|_{-\alpha}^{2} & =\mathbb{E}\left\|\int_{\mathbf{R}}\left(A^{y}(t)-A^{y}(s)\right) \mu(d y)\right\|_{-\alpha}^{2} \\
& \leq C \int_{\mathbf{R}} \mathbb{E}\left\|A^{y}(t)-A^{y}(s)\right\|_{-\alpha}^{2}|\mu|(d y) \\
& \leq C \varepsilon|t-s|^{\delta} \int_{\mathbf{R}}\left|y^{3}\right||\mu|(d y) \leq C \varepsilon|t-s|^{\delta},
\end{aligned}
$$

which is the desired bound.

The result follows by combining these steps with Lemma 4.1 .

5. Convergence of the approximations. This last section is devoted to the convergence result itself. Recall that we are considering a number of intermediate processes $v_{\varepsilon}^{(j)}$ with $j=1, \ldots, 4$ defined in $(2.10)$. This section is correspondingly broken into five subsections, with the $j$ th subsection yielding a bound on $\left\|v_{\varepsilon}^{(j)}-v_{\varepsilon}^{(j-1)}\right\|_{\alpha}$. To prove these bounds, we shall introduce in each step a stopping time that forces the difference between the processes considered in that step to remain bounded. We then show that this difference actually vanishes as $\varepsilon \rightarrow 0$ with an explicit rate. As a consequence, the process actually does not "see" the stopping time with high probability.

\subsection{From $\bar{v}^{\gamma}$ to $v_{\varepsilon}^{(1)}$. Define}

$$
\tau_{1}^{K}:=\tau^{K} \wedge \inf \left\{t \leq T:\left\|v_{\varepsilon}^{(1)}(t)-\bar{v}^{\gamma}(t)\right\|_{\alpha} \geq K\right\} .
$$

We shall show that for $t \leq \tau_{K}$, the $H^{\alpha}$-norm of $v_{\varepsilon}^{(1)}(t)-\bar{v}^{\gamma}(t)$ is controlled by the $L^{\infty}$-norm of $\psi_{\gamma}$, which is of order $\varepsilon^{\gamma / 2-\kappa}$ for any $\kappa>0$, as shown in Section 4. The proof uses the mild formulations of the equations for $v_{\varepsilon}^{(1)}$ and $\bar{v}^{\gamma}(t)$ as well as the regularizing properties of the semigroup $S$. Note 
that the next proposition would still be true if we had replaced the $H^{\alpha_{-}}$ norm in the definition of $\tau_{1}^{K}$ by the $L^{\infty}$-norm. However, in the proof of Proposition 5.2 below it will be important to have a bound on $v_{\varepsilon}^{(1)}$ in $H^{\alpha}$.

Proposition 5.1. For $\kappa>0$, we have

$$
\lim _{\varepsilon \rightarrow 0} \mathbb{P}\left(\sup _{t \leq \tau_{1}^{K}}\left\|v_{\varepsilon}^{(1)}(t)-\bar{v}^{\gamma}(t)\right\|_{\alpha}>\varepsilon^{\gamma / 2-\kappa}\right)=0 .
$$

Proof. Let $0 \leq s \leq t \leq \tau^{*}$. It follows from (2.3) and (2.10a) that $\varrho_{\varepsilon}:=$ $v_{\varepsilon}^{(1)}-\bar{v}^{\gamma}$ satisfies the equation

$$
\varrho_{\varepsilon}(t)=S(t-s) \varrho_{\varepsilon}(s)+\int_{s}^{t} S(t-r)\left(\sigma^{1}+\partial_{x} \sigma_{\varepsilon}^{2}\right)(r) d r
$$

where

$$
\begin{aligned}
\sigma_{\varepsilon}^{1} & :=\bar{F}\left(\bar{v}^{\gamma}+\varrho_{\varepsilon}\right)-\bar{F}\left(\bar{v}^{\gamma}+\psi_{\gamma}\right), \\
\sigma_{\varepsilon}^{2} & :=G\left(\bar{v}^{\gamma}+\varrho_{\varepsilon}\right)-G\left(\bar{v}^{\gamma}+\psi_{\gamma}\right) .
\end{aligned}
$$

Lemma 3.3 yields the estimate

$$
\begin{aligned}
\left\|\varrho_{\varepsilon}(t)\right\|_{\alpha} & \leq\left\|\varrho_{\varepsilon}(s)\right\|_{\alpha}+C(t-s)^{(1-\alpha) / 2} \sup _{r \in(s, t)}\left\|\left(\sigma_{\varepsilon}^{1}+\partial_{x} \sigma_{\varepsilon}^{2}\right)(r)\right\|_{-1} \\
& \leq\left\|\varrho_{\varepsilon}(s)\right\|_{\alpha}+C(t-s)^{(1-\alpha) / 2} \sup _{r \in(s, t)}\left\|\sigma_{\varepsilon}^{1}(r)\right\|_{L^{\infty}}+\left\|\sigma_{\varepsilon}^{2}(r)\right\|_{L^{\infty}} .
\end{aligned}
$$

Since $\bar{v}^{\gamma}, \varrho_{\varepsilon}$, and $\psi_{\gamma}$ are bounded in $L^{\infty}$-norm for $r \leq \tau_{1}^{K}$, and $F, G$ are $\mathcal{C}^{3}$, it follows that

$$
\left\|\sigma_{\varepsilon}^{1}(r)\right\|_{L^{\infty}}+\left\|\sigma_{\varepsilon}^{2}(r)\right\|_{L^{\infty}} \leq C_{K}\left\|\varrho_{\varepsilon}(r)\right\|_{L^{\infty}}+C_{K}\left\|\psi_{\gamma}(r)\right\|_{L^{\infty}},
$$

from which we infer that

$$
\left\|\varrho_{\varepsilon}(t)\right\|_{\alpha} \leq\left\|\varrho_{\varepsilon}(s)\right\|_{\alpha}+C_{K}^{\prime}(t-s)^{(1-\alpha) / 2} \sup _{r \in(s, t)}\left(\left\|\varrho_{\varepsilon}(r)\right\|_{L^{\infty}}+\left\|\psi_{\gamma}(r)\right\|_{L^{\infty}}\right) .
$$

Choose $\delta_{K}>0$ so small that $C_{K}^{\prime} \delta_{K}^{(1-\alpha) / 2} \leq \frac{1}{2}$, and set for $k \geq 0$,

$$
r_{k}:=\sup \left\{\left\|\varrho_{\varepsilon}(t)\right\|_{\alpha}: t \in\left[k \delta_{K} \wedge \tau_{1}^{K},(k+1) \delta_{K} \wedge \tau_{1}^{K}\right]\right\} .
$$

Taking into account that $H^{\alpha} \subseteq L^{\infty}$, we obtain the inequality

$$
r_{k+1} \leq r_{k}+\frac{1}{2} r_{k+1}+\frac{1}{2} \sup _{t \in[0, T]}\left\|\psi_{\gamma}(t)\right\|_{L^{\infty}},
$$

which reduces to

$$
r_{k+1} \leq 2 r_{k}+\sup _{t \in[0, T]}\left\|\psi_{\gamma}(t)\right\|_{L^{\infty}} .
$$

Combined with the estimate

$$
r_{0} \leq 2 \sup _{r \leq \delta_{K} \wedge \tau_{1}^{K}}\left\|\psi_{\gamma}(r)\right\|_{L^{\infty}}
$$


which can be derived similarly, it then follows that

$$
\sup _{t \in\left[0, \tau_{1}^{K}\right]}\left\|\varrho_{\varepsilon}(t)\right\|_{\alpha} \leq \sup _{0 \leq k \leq T / \delta_{K}} r_{k} \leq C_{K} \sup _{t \in[0, T]}\left\|\psi_{\gamma}(t)\right\|_{L^{\infty}},
$$

which together with Proposition 4.3 implies the desired result.

5.2. From $v_{\varepsilon}^{(1)}$ to $v_{\varepsilon}^{(2)}$. For the purpose of this section, we define the stopping time

$$
\tau_{2}^{K}:=\tau_{1}^{K} \wedge \inf \left\{t \leq T:\left\|v_{\varepsilon}^{(2)}(t)-v_{\varepsilon}^{(1)}(t)\right\|_{\alpha} \geq K\right\}
$$

as well as the exponent

$$
\widetilde{\alpha} \stackrel{\text { def }}{=}(1-\gamma) \alpha+\frac{\gamma}{2}=\frac{2}{3} .
$$

Proposition 5.2. For $\kappa>0$, we have

$$
\lim _{\varepsilon \rightarrow 0} \mathbb{P}\left(\sup _{t \leq \tau_{2}^{K}}\left\|v_{\varepsilon}^{(2)}(t)-v_{\varepsilon}^{(1)}(t)\right\|_{\alpha}>\varepsilon^{\widetilde{\alpha}-\kappa}\right)=0 .
$$

Proof. Let $0 \leq s \leq t \leq \tau^{*}$ and note that $\varrho_{\varepsilon}:=v_{\varepsilon}^{(2)}-v_{\varepsilon}^{(1)}$ satisfies

$$
\varrho_{\varepsilon}(t)=S(t-s) \varrho_{\varepsilon}(s)+\int_{s}^{t} S(t-r) \sigma_{\varepsilon}(r) d r,
$$

where

$$
\sigma_{\varepsilon}:=\bar{F}\left(v_{\varepsilon}^{(2)}\right)-\bar{F}\left(v_{\varepsilon}^{(1)}\right)+D_{\varepsilon}\left(G\left(v_{\varepsilon}^{(1)}+\varrho_{\varepsilon}\right)\right)-\partial_{x} G\left(v_{\varepsilon}^{(1)}\right) .
$$

From the definition of $\tau_{2}^{K}$, we know that $v_{\varepsilon}^{(1)}$ and $\varrho_{\varepsilon}$ are bounded in $L^{\infty}$ by a constant depending on $K$. Moreover, we have the bound $\left\|v_{\varepsilon}^{(1)}\right\|_{\alpha} \leq$ $C_{K} \varepsilon^{-\gamma(\alpha-1 / 2)-\kappa}$. Using these facts together with Corollary 3.7 we obtain, for $r \leq \tau_{2}^{K}$,

$$
\begin{aligned}
\left\|\sigma_{\varepsilon}\right\|_{-1} \leq & \left\|\bar{F}\left(v_{\varepsilon}^{(2)}\right)-\bar{F}\left(v_{\varepsilon}^{(1)}\right)\right\|_{L^{\infty}} \\
& +\left\|\left(D_{\varepsilon}-\partial_{x}\right) G\left(v_{\varepsilon}^{(1)}\right)\right\|_{-1}+\left\|D^{\varepsilon}\left(G\left(v_{\varepsilon}^{(1)}+\varrho_{\varepsilon}\right)-G\left(v_{\varepsilon}^{(1)}\right)\right)\right\|_{-1} \\
\leq & C_{K}\left\|\varrho_{\varepsilon}\right\|_{L^{\infty}}+C_{K} \varepsilon^{\alpha}\left(1+\left\|v_{\varepsilon}^{(1)}\right\|_{\alpha}\right)+\left\|G\left(v_{\varepsilon}^{(1)}+\varrho_{\varepsilon}\right)-G\left(v_{\varepsilon}^{(1)}\right)\right\|_{L^{\infty}} \\
\leq & C_{K}\left(\varepsilon^{\widetilde{\alpha}-\kappa}+\left\|\varrho_{\varepsilon}\right\|_{L^{\infty}}\right),
\end{aligned}
$$

hence

$$
\begin{aligned}
\left\|\varrho_{\varepsilon}(t)\right\|_{\alpha} & \leq\left\|\varrho_{\varepsilon}(s)\right\|_{\alpha}+C(t-s)^{(1-\alpha) / 2} \sup _{r \in(s, t)}\left\|\sigma_{\varepsilon}(r)\right\|_{-1} \\
& \leq\left\|\varrho_{\varepsilon}(s)\right\|_{\alpha}+C_{K}(t-s)^{(1-\alpha) / 2} \sup _{r \in(s, t)}\left(\varepsilon^{\widetilde{\alpha}-\kappa}+\left\|\varrho_{\varepsilon}(r)\right\|_{L^{\infty}}\right) .
\end{aligned}
$$


Arguing as in the proof of Proposition 5.1, it follows that

$$
\sup _{t \in\left[0, \tau_{2}^{K}\right]}\left\|\varrho_{\varepsilon}(t)\right\|_{\alpha} \leq C_{K} \varepsilon^{\widetilde{\alpha}-\kappa}
$$

which immediately yields the desired result.

5.3. From $v_{\varepsilon}^{(2)}$ to $v_{\varepsilon}^{(3)}$. Define

$$
\tau_{3}^{K}:=\tau_{2}^{K} \wedge \inf \left\{t \leq T:\left\|v_{\varepsilon}^{(3)}(t)-v_{\varepsilon}^{(2)}(t)\right\|_{\alpha} \geq K\right\} .
$$

In this case, the singularity $(t-s)^{-\alpha / 2}$ which arises in the proof below, prevents us from arguing as in Proposition 5.1. We nevertheless have the following proposition.

Proposition 5.3. For $\kappa>0$, we have

$$
\lim _{\varepsilon \rightarrow 0} \mathbb{P}\left(\sup _{t \leq \tau_{3}^{K}}\left\|v_{\varepsilon}^{(3)}(t)-v_{\varepsilon}^{(2)}(t)\right\|_{\alpha}>\varepsilon^{\zeta-\kappa}\right)=0,
$$

where the exponent $\zeta$ is given by

$$
\zeta \stackrel{\text { def }}{=} \widetilde{\alpha} \wedge\left(\frac{3}{2}-\alpha\right) \wedge\left(2-\gamma\left(\alpha+\frac{3}{2}\right)\right)=\frac{2}{3} .
$$

REMARK 5.4. The exponent $\zeta$ arises by collecting the bounds (5.2), (5.3), and (5.5).

Proof of Proposition 5.3. Let $0 \leq s \leq t \leq \tau^{*}$. It follows from (2.10b) and $(2.10 \mathrm{c})$ that $\varrho_{\varepsilon}:=v_{\varepsilon}^{(3)}-v_{\varepsilon}^{(2)}$ satisfies

$$
\begin{aligned}
\varrho_{\varepsilon}(t)= & S_{\varepsilon}(t-s) \varrho_{\varepsilon}(s)+\left(S_{\varepsilon}(t-s)-S(t-s)\right) v_{\varepsilon}^{(2)}(s) \\
& +\mathcal{R}_{1}(s, t)+\mathcal{R}_{2}(s, t),
\end{aligned}
$$

where

$$
\mathcal{R}_{1}(s, t) \stackrel{\text { def }}{=}\left(\widetilde{\psi}^{\gamma}(t)-\psi^{\gamma}(t)\right)-\left(S_{\varepsilon}(t-s) \widetilde{\psi}^{\gamma}(s)-S(t-s) \psi^{\gamma}(s)\right)
$$

and

$$
\begin{aligned}
\mathcal{R}_{2}(s, t):=\int_{s}^{t}\left(S_{\varepsilon}(t-r)-\right. & S(t-r))\left(\bar{F}\left(v_{\varepsilon}^{(3)}(r)\right)+D_{\varepsilon} G\left(v_{\varepsilon}^{(3)}(r)\right)\right) d r \\
& +\int_{s}^{t} S(t-r)\left(\bar{F}\left(v_{\varepsilon}^{(3)}(r)\right)-\bar{F}\left(v_{\varepsilon}^{(2)}(r)\right)\right. \\
& \left.+D_{\varepsilon} G\left(v_{\varepsilon}^{(3)}(r)\right)-D_{\varepsilon} G\left(v_{\varepsilon}^{(2)}(r)\right)\right) d r .
\end{aligned}
$$

We shall first prove a bound on $\mathcal{R}_{1}(s, t)$. Using both inequalities from Lemma 3.4, we obtain

$$
\begin{aligned}
& \left\|\left(S_{\varepsilon}(t-s) \widetilde{\psi}^{\gamma}(s)-S(t-s) \psi^{\gamma}(s)\right)\right\|_{\alpha} \\
& \quad \leq\left\|S_{\varepsilon}(t-s)\left(\widetilde{\psi}^{\gamma}(s)-\psi^{\gamma}(s)\right)\right\|_{\alpha}+\left\|\left(S_{\varepsilon}(t-s)-S(t-s)\right) \psi^{\gamma}(s)\right\|_{\alpha} \\
& \quad \leq C\left\|\left(\widetilde{\psi}^{\gamma}(s)-\psi^{\gamma}(s)\right)\right\|_{\alpha}+C \varepsilon^{2}\left\|\psi^{\gamma}(s)\right\|_{\alpha+2},
\end{aligned}
$$


and therefore

$$
\begin{aligned}
\left\|\mathcal{R}_{1}(s, t)\right\|_{\alpha} \leq & \left\|\left(\widetilde{\psi}^{\gamma}(t)-\psi^{\gamma}(t)\right)\right\|_{\alpha}+C\left\|\left(\widetilde{\psi}^{\gamma}(s)-\psi^{\gamma}(s)\right)\right\|_{\alpha} \\
& +C \varepsilon^{2}\left\|\psi^{\gamma}(s)\right\|_{\alpha+2} .
\end{aligned}
$$

It thus follows from Proposition 4.4 that

$$
\mathbb{E} \sup _{s, t \in[0, T]}\left\|\mathcal{R}_{1}(s, t)\right\|_{\alpha} \leq C \varepsilon^{2-\gamma(\alpha+3 / 2)-\kappa} .
$$

We shall now prove a bound on $\mathcal{R}_{2}(s, t)$. For this purpose, we note that the definitions of the various stopping times imply that $v_{\varepsilon}^{(2)}(t)$ is bounded in $H^{\alpha}$-norm by $C_{K} \varepsilon^{-\gamma(\alpha-1 / 2)-\kappa}$. Using this fact, together with Lemmas 3.4, 3.5 and 3.2 , we obtain

$$
\begin{aligned}
& \left\|\int_{s}^{t}\left(S_{\varepsilon}(t-r)-S(t-r)\right)\left(F\left(v_{\varepsilon}^{(3)}(r)\right)+D_{\varepsilon} G\left(v_{\varepsilon}^{(3)}(r)\right)\right) d r\right\|_{\alpha} \\
& \quad \leq \varepsilon^{\alpha} \int_{s}^{t}(t-r)^{-(1+\alpha) / 2}\left\|F\left(v_{\varepsilon}^{(3)}(r)\right)+D_{\varepsilon} G\left(v_{\varepsilon}^{(3)}(r)\right)\right\|_{\alpha-1} d r \\
& \quad \leq C \varepsilon^{\alpha}(t-s)^{(1-\alpha) / 2} \sup _{r \in[s, t]}\left(\left\|F\left(v_{\varepsilon}^{(3)}(r)\right)\right\|_{\alpha}+\left\|G\left(v_{\varepsilon}^{(3)}(r)\right)\right\|_{\alpha}\right) \\
& \quad \leq C_{K} \varepsilon^{\alpha}(t-s)^{(1-\alpha) / 2}\left(1+\sup _{r \in[s, t]}\left\|v_{\varepsilon}^{(3)}(r)\right\|_{\alpha}\right) \\
& \quad \leq C_{K} \varepsilon^{\alpha}(t-s)^{(1-\alpha) / 2}\left(1+\sup _{r \in[s, t]}\left(\left\|v_{\varepsilon}^{(2)}(r)\right\|_{\alpha}+\left\|\varrho_{\varepsilon}(r)\right\|_{\alpha}\right)\right) \\
& \quad \leq C_{K} \varepsilon^{\widetilde{\alpha}-\kappa}(t-s)^{(1-\alpha) / 2} .
\end{aligned}
$$

Furthermore, taking into account the $L^{\infty}$-bounds on $v_{\varepsilon}^{(2)}$ and $\varrho_{\varepsilon}$ enforced by the stopping times, Lemma 3.5 implies that

$$
\begin{gathered}
\left\|\int_{s}^{t} S(t-r)\left(F\left(v_{\varepsilon}^{(3)}(r)\right)-F\left(v_{\varepsilon}^{(2)}(r)\right)+D_{\varepsilon} G\left(v_{\varepsilon}^{(3)}(r)\right)-D_{\varepsilon} G\left(v_{\varepsilon}^{(2)}(r)\right)\right) d r\right\|_{\alpha} \\
\leq C(t-s)^{(1-\alpha) / 2} \sup _{r \in(s, t)} \| F\left(v_{\varepsilon}^{(3)}(r)\right)-F\left(v_{\varepsilon}^{(2)}(r)\right) \\
\quad+D_{\varepsilon} G\left(v_{\varepsilon}^{(3)}(r)\right)-D_{\varepsilon} G\left(v_{\varepsilon}^{(2)}(r)\right) \|_{-1} \\
\leq C(t-s)^{(1-\alpha) / 2} \sup _{r \in(s, t)}\left(\left\|F\left(v_{\varepsilon}^{(3)}(r)\right)-F\left(v_{\varepsilon}^{(2)}(r)\right)\right\|_{L^{\infty}}\right. \\
\left.\quad+\left\|G\left(v_{\varepsilon}^{(3)}(r)\right)-G\left(v_{\varepsilon}^{(2)}(r)\right)\right\|_{L^{\infty}}\right) \\
\leq C_{K}(t-s)^{(1-\alpha) / 2} \sup _{r \in(s, t)}\left\|\varrho_{\varepsilon}(r)\right\|_{L^{\infty}} .
\end{gathered}
$$


It thus follows that

$$
\left\|\mathcal{R}_{2}(s, t)\right\|_{\alpha} \leq C_{K}^{\prime}(t-s)^{(1-\alpha) / 2}\left(\varepsilon^{\widetilde{\alpha}-\kappa}+\sup _{r \in(s, t)}\left\|\varrho_{\varepsilon}(r)\right\|_{L^{\infty}}\right),
$$

where we gave the constant a name, since it will be reused below.

Choose $\delta_{K} \in(0,1)$ sufficiently small so that $C_{K}^{\prime}\left(\delta_{K}^{(1-\alpha) / 2}+\delta_{K}^{\alpha / 2}\right) \leq \frac{1}{4}$. For $k \geq 0$ put $\ell_{k}:=k \delta_{K} \wedge \tau_{3}^{K}$, and for $k \geq 1$ set

$$
r_{k}:=\sup \left\{\left\|\varrho_{\varepsilon}(t)\right\|_{\alpha}: t \in\left[\ell_{k-1}, \ell_{k+1}\right]\right\} \text {. }
$$

Our next aim is to find a bound for $r_{1}$. Observe that, when $s=0,(5.1)$ simplifies to

$$
\varrho_{\varepsilon}(t)=\left(S_{\varepsilon}(t)-S(t)\right) v_{0}+\left(\widetilde{\psi}^{\gamma}(t)-\psi^{\gamma}(t)\right)+\mathcal{R}_{2}(0, t)
$$

with $\mathcal{R}_{2}$ defined as previously. Using Lemma 3.4 and the definition of $\tau^{K}$, we obtain

$$
\left\|\left(S_{\varepsilon}(t)-S(t)\right) v_{0}\right\|_{\alpha} \leq C \varepsilon^{3 / 2-\alpha-\kappa}\left\|v_{0}\right\|_{3 / 2-\kappa} \leq C_{K} \varepsilon^{3 / 2-\alpha-\kappa} .
$$

Since $t \leq 2 \delta_{K}$ and $C_{K}^{\prime} \delta_{K}^{(1-\alpha) / 2} \leq \frac{1}{4}$, it follows from (5.3) and (5.4) that

$$
r_{1} \leq C_{K} \varepsilon^{3 / 2-\alpha-\kappa}+\sup _{t \in[0, T]}\left\|\widetilde{\psi}^{\gamma}(t)-\psi^{\gamma}(t)\right\|_{\alpha}+\frac{1}{2}\left(\varepsilon^{\widetilde{\alpha}-\kappa}+r_{1}\right),
$$

hence, by definition of $\tau^{K}$,

$$
r_{1} \leq C_{K} \varepsilon^{(3 / 2-\alpha) \wedge(2-\gamma(\alpha+3 / 2)) \wedge \widetilde{\alpha}} \varepsilon^{-\kappa}=C_{K} \varepsilon^{\zeta-\kappa},
$$

where $\zeta$ is defined as in the statement of the result.

Next, we shall prove a recursive bound for $r_{k}$. Note that the nonnegativity of the function $f$ in the definition of $S_{\varepsilon}$ implies that

$$
\left\|S_{\varepsilon}(t-s) \varrho_{\varepsilon}(s)\right\|_{\alpha} \leq\left\|\varrho_{\varepsilon}(s)\right\|_{\alpha} .
$$

Furthermore, by Lemma 3.4 and the fact that $\left\|v_{\varepsilon}^{(2)}\right\|_{\alpha} \leq C_{K} \varepsilon^{-\gamma(\alpha-1 / 2)-\kappa}$ before time $\tau_{3}^{K}$, we have

$$
\begin{aligned}
\left\|\left(S_{\varepsilon}(t-s)-S(t-s)\right) v_{\varepsilon}^{(2)}(s)\right\|_{\alpha} & \leq C \varepsilon^{\alpha}(t-s)^{-\alpha / 2}\left\|v_{\varepsilon}^{(2)}(s)\right\|_{\alpha} \\
& \leq C_{K}(t-s)^{-\alpha / 2} \varepsilon^{\widetilde{\alpha}-\kappa} .
\end{aligned}
$$

Combining these bounds with (5.1) and (5.3), we find that

$$
\begin{aligned}
\left\|\varrho_{\varepsilon}(t)\right\|_{\alpha} \leq & \left\|\varrho_{\varepsilon}(s)\right\|_{\alpha}+C_{K}(t-s)^{-\alpha / 2} \varepsilon^{\widetilde{\alpha}-\kappa}+\left\|\mathcal{R}_{1}(s, t)\right\|_{\alpha} \\
& +C_{K}^{\prime}(t-s)^{(1-\alpha) / 2}\left(\varepsilon^{\widetilde{\alpha}-\kappa}+\sup _{r \in(s, t)}\left\|\varrho_{\varepsilon}(r)\right\|_{\alpha}\right) .
\end{aligned}
$$

Taking $k \geq 1, s=\ell_{k-1}$, and $t \in\left[\ell_{k}, \ell_{k+2}\right]$, it then follows, since $|t-s| \in$ $\left[\delta_{K}, 3 \delta_{K}\right]$ and $C_{K}^{\prime} \delta^{(1-\alpha) / 2} \leq \frac{1}{2}$, that

$$
\left\|\varrho_{\varepsilon}(t)\right\|_{\alpha} \leq\left\|\varrho_{\varepsilon}\left(\ell_{k-1}\right)\right\|_{\alpha}+C_{K} \varepsilon^{\widetilde{\alpha}-\kappa}+\left\|\mathcal{R}_{1}\left(\ell_{k-1}, t\right)\right\|_{\alpha}+\frac{1}{2} \varepsilon^{\widetilde{\alpha}-\kappa}+\frac{1}{2} r_{k+1} .
$$


Taking the supremum over $t \in\left[\ell_{k}, \ell_{k+2}\right]$, we obtain

$$
r_{k+1} \leq r_{k}+C_{K} \varepsilon^{\widetilde{\alpha}-\kappa}+\sup _{s, t \in[0, T]}\left\|\mathcal{R}_{1}(s, t)\right\|_{\alpha}+\frac{1}{2} r_{k+1},
$$

hence

$$
r_{k+1} \leq 2 r_{k}+C_{K} \varepsilon^{\widetilde{\alpha}-\kappa}+2 \sup _{s, t \in[0, T]}\left\|\mathcal{R}_{1}(s, t)\right\|_{\alpha} .
$$

It readily follows from (5.6) and (5.7) that

$$
\sup _{t \in\left[0, \tau_{3}^{K}\right]}\left\|\varrho_{\varepsilon}(t)\right\|_{\alpha}=\sup _{1 \leq k \leq\left\lceil T / \delta_{K}\right\rceil} r_{k} \leq C_{K}\left(\varepsilon^{\zeta-\kappa}+\varepsilon^{\widetilde{\alpha}-\kappa}+\sup _{s, t \in[0, T]}\left\|\mathcal{R}_{1}(s, t)\right\|_{\alpha}\right),
$$

hence the result follows in view of the bound on $\mathcal{R}_{1}(s, t)$.

5.4. From $v_{\varepsilon}^{(3)}$ to $v_{\varepsilon}^{(4)}$. Define

$$
\tau_{4}^{K}:=\tau_{3}^{K} \wedge \inf \left\{t \leq T:\left\|v_{\varepsilon}^{(4)}(t)-v_{\varepsilon}^{(3)}(t)\right\|_{\alpha} \geq K\right\} .
$$

Proposition 5.5. For $\kappa>0$, we have

$$
\lim _{\varepsilon \rightarrow 0} \mathbb{P}\left(\sup _{t \leq \tau_{4}^{K}}\left\|v_{\varepsilon}^{(4)}(t)-v_{\varepsilon}^{(3)}(t)\right\|_{\alpha}>\varepsilon^{\xi-\kappa}\right)=0,
$$

where

$$
\xi \stackrel{\text { def }}{=} \frac{\gamma}{2} \wedge\left(\widetilde{\alpha}-\frac{1}{2}\right) \wedge\left(\frac{1}{2}-\chi\left(\alpha-\frac{1}{2}\right)\right)=\frac{1}{8} .
$$

REMARK 5.6. Similarly to above, the exponent $\xi$ arises from the bounds (5.9)-(5.14).

Proof of Proposition 5.5. Let $0 \leq s \leq t \leq \tau^{*}$. It follows from (2.10d) and $(2.10 \mathrm{c})$ that $\varrho_{\varepsilon}:=v_{\varepsilon}^{(4)}-v_{\varepsilon}^{(3)}$ satisfies

$$
\varrho_{\varepsilon}(t)=S_{\varepsilon}(t-s) \varrho_{\varepsilon}(s)+\int_{s}^{t} S_{\varepsilon}(t-r) \sigma_{\varepsilon}(r) d r
$$

where

$$
\begin{aligned}
\sigma_{\varepsilon}:= & F\left(v_{\varepsilon}^{(4)}+\widetilde{\psi}_{\gamma}^{\chi}\right)-F\left(v_{\varepsilon}^{(3)}\right) \\
& +\nabla G\left(v_{\varepsilon}^{(4)}+\widetilde{\psi}_{\gamma}^{\chi}\right) D_{\varepsilon}\left(v_{\varepsilon}^{(4)}+\widetilde{\psi}_{\gamma}^{\chi}\right)-D_{\varepsilon} G\left(v_{\varepsilon}^{(3)}\right)+\Lambda \Delta G\left(v_{\varepsilon}^{(3)}\right) .
\end{aligned}
$$

The definition of $D_{\varepsilon}$, together with (1.3), implies that for any function $u$ the following identity holds:

$$
\begin{aligned}
& D_{\varepsilon} G(u)(x) \\
& \quad=\nabla G(u(x)) D_{\varepsilon} u(x)
\end{aligned}
$$




$$
\begin{aligned}
& +\int_{\mathbf{R}} \frac{\varepsilon y^{2}}{2} D^{2} G(u(x))\left[\widehat{D}_{\varepsilon y} u(x), \widehat{D}_{\varepsilon y} u(x)\right] \mu(d y) \\
& +\int_{\mathbf{R}} \varepsilon^{2} y^{3} \int_{0}^{1} \int_{0}^{t} \int_{0}^{s} D^{3} G((1-r) u(x)+r u(x+\varepsilon y)) \\
& \times\left[\widehat{D}_{\varepsilon y} u(x), \widehat{D}_{\varepsilon y} u(x), \widehat{D}_{\varepsilon y} u(x)\right] d r d s d t \mu(d y),
\end{aligned}
$$

where the operator $\widehat{D}_{\varepsilon}$ is defined by taking $\mu:=\delta_{1}-\delta_{0}$ in the definition of $D_{\varepsilon}$, that is, $\widehat{D}_{\varepsilon} u(x)=\varepsilon^{-1}(u(x+\varepsilon)-u(x))$. As a consequence, we may write

$$
\begin{aligned}
\sigma_{\varepsilon}= & F\left(v_{\varepsilon}^{(4)}+\widetilde{\psi}_{\gamma}^{\chi}\right)-F\left(v_{\varepsilon}^{(3)}\right)+D_{\varepsilon}\left(G\left(v_{\varepsilon}^{(4)}+\widetilde{\psi}_{\gamma}^{\chi}\right)-G\left(v_{\varepsilon}^{(3)}\right)\right) \\
& +\left(\Lambda \Delta G\left(v_{\varepsilon}^{(3)}\right)-A\left(u_{\varepsilon}^{(4)}, u_{\varepsilon}^{(4)}\right)\right)-B \\
= & F\left(v_{\varepsilon}^{(4)}+\widetilde{\psi}_{\gamma}^{\chi}\right)-F\left(v_{\varepsilon}^{(3)}\right)+D_{\varepsilon}\left(G\left(v_{\varepsilon}^{(4)}+\widetilde{\psi}_{\gamma}^{\chi}\right)-G\left(v_{\varepsilon}^{(3)}\right)\right) \\
& -A\left(v_{\varepsilon}^{(4)}, v_{\varepsilon}^{(4)}\right)-2 A\left(v_{\varepsilon}^{(4)}, \widetilde{\psi}_{\gamma}^{\chi}\right)+\left(\Lambda \Delta G\left(v_{\varepsilon}^{(3)}\right)-A\left(\widetilde{\psi}_{\gamma}^{\chi}, \widetilde{\psi}_{\gamma}^{\chi}\right)\right)-B,
\end{aligned}
$$

where we have used

$$
\begin{aligned}
A(v, w)(x) \stackrel{\text { def }}{=} \int_{\mathbf{R}} \frac{\varepsilon y^{2}}{2} D^{2} G\left(u_{\varepsilon}^{(4)}(x)\right)\left[\widehat{D}_{\varepsilon y} v(x), \widehat{D}_{\varepsilon y} w(x)\right] \mu(d y), \\
B(x) \stackrel{\text { def }}{=} \int_{\mathbf{R}} \varepsilon^{2} y^{3} \int_{0}^{1} \int_{0}^{t} \int_{0}^{s} D^{3} G\left((1-r) u_{\varepsilon}^{(4)}(x)+r u_{\varepsilon}^{(4)}(x+\varepsilon y)\right) \\
\times\left[\widehat{D}_{\varepsilon y} u_{\varepsilon}^{(4)}(x), \widehat{D}_{\varepsilon y} u_{\varepsilon}^{(4)}(x),\right. \\
\left.\widehat{D}_{\varepsilon y} u_{\varepsilon}^{(4)}(x)\right] d r d s d t \mu(d y)
\end{aligned}
$$

and $u_{\varepsilon}^{(4)}:=v_{\varepsilon}^{(4)}+\widetilde{\psi}_{\gamma}^{\chi}$.

Our next aim is to prove the estimates (5.9)-(5.14) below in order to bound $\left\|\sigma_{\varepsilon}\right\|_{-1}$.

First term. Since $v_{\varepsilon}^{(4)}, \widetilde{\psi}_{\gamma}^{\chi}$ and $\varrho_{\varepsilon}$ are bounded in $L^{\infty}$ by definition of $\tau_{4}^{K}$, it follows that

$$
\begin{aligned}
\left\|F\left(v_{\varepsilon}^{(4)}+\widetilde{\psi}_{\gamma}^{\chi}\right)-F\left(v_{\varepsilon}^{(3)}\right)\right\|_{-1} & \leq C\left\|F\left(v_{\varepsilon}^{(4)}+\widetilde{\psi}_{\gamma}^{\chi}\right)-F\left(v_{\varepsilon}^{(4)}-\varrho_{\varepsilon}\right)\right\|_{L^{\infty}} \\
& \leq C_{K}\left(\left\|\widetilde{\psi}_{\gamma}^{\chi}\right\|_{L^{\infty}}+\left\|\varrho_{\varepsilon}\right\|_{L^{\infty}}\right) \\
& \leq C_{K}\left(\varepsilon^{\gamma / 2-\kappa}+\left\|\varrho_{\varepsilon}\right\|_{\alpha}\right) .
\end{aligned}
$$

Second term. We use Lemma 3.5 and the fact that $v_{\varepsilon}^{(4)}, \widetilde{\psi}_{\gamma}^{\chi}$ and $\varrho_{\varepsilon}$ are bounded in $L^{\infty}$ by definition of $\tau_{4}^{K}$, to estimate

$$
\begin{aligned}
\left\|D_{\varepsilon}\left(G\left(v_{\varepsilon}^{(4)}+\widetilde{\psi}_{\gamma}^{\chi}\right)-G\left(v_{\varepsilon}^{(3)}\right)\right)\right\|_{-1} & \leq C\left\|G\left(v_{\varepsilon}^{(4)}+\widetilde{\psi}_{\gamma}^{\chi}\right)-G\left(v_{\varepsilon}^{(4)}-\varrho_{\varepsilon}\right)\right\|_{L^{\infty}} \\
& \leq C_{K}\left(\left\|\widetilde{\psi}_{\gamma}^{\chi}\right\|_{L^{\infty}}+\left\|\varrho_{\varepsilon}\right\|_{L^{\infty}}\right) \\
& \leq C_{K}\left(\varepsilon^{\gamma / 2-\kappa}+\left\|\varrho_{\varepsilon}\right\|_{\alpha}\right) .
\end{aligned}
$$


Third and fourth term. First, we note that for arbitrary functions $v, w$, one has

$$
\|A(v, w)\|_{-1} \leq C\|A(v, w)\|_{L^{1}} \leq C \varepsilon\left\|D^{2} G\left(u_{\varepsilon}^{(4)}\right)\right\|_{L^{\infty}} \sqrt{\Theta_{\varepsilon}(v) \Theta_{\varepsilon}(w)} .
$$

Since $\left\|u_{\varepsilon}^{(4)}(t)\right\|_{L^{\infty}} \leq C_{K}$ for $t \leq \tau_{4}^{K}$, we have

$$
\left\|D^{2} G\left(u_{\varepsilon}^{(4)}\right)\right\|_{L^{\infty}} \leq C_{K} .
$$

Furthermore, we observe that $\left\|v_{\varepsilon}^{(4)}\right\|_{\alpha} \leq C_{K} \varepsilon^{-\gamma(\alpha-1 / 2)-\kappa}$ before time $\tau_{4}^{K}$. Using this bound together with Lemma 3.5 and (1.3), we estimate

$$
\begin{aligned}
\Theta_{\varepsilon}\left(v_{\varepsilon}^{(4)}\right) & =\int_{\mathbf{R}} y^{2}\left\|\widehat{D}_{\varepsilon y} v_{\varepsilon}^{(4)}\right\|_{L^{2}}^{2}|\mu|(d y) \\
& \leq C_{K} \int_{\mathbf{R}} y^{2}|\varepsilon y|^{2(\alpha-1)}\left\|v_{\varepsilon}^{(4)}\right\|_{\alpha}^{2}|\mu|(d y) \\
& \leq C_{K} \int_{\mathbf{R}} y^{2}|\varepsilon y|^{2(\alpha-1)} \varepsilon^{-2 \gamma(\alpha-1 / 2)-2 \kappa}|\mu|(d y) \\
& \leq C_{K} \varepsilon^{2 \widetilde{\alpha}-2-\kappa} .
\end{aligned}
$$

Moreover, by definition of the stopping time $\tau^{K}$ we have

$$
\Theta_{\varepsilon}\left(\widetilde{\psi}_{\gamma}^{\chi}\right) \leq \Theta_{\varepsilon}\left(\widetilde{\psi}_{\gamma}\right) \leq C_{K} \varepsilon^{-1-\kappa} .
$$

Putting everything together, we obtain

$$
\left\|A\left(v_{\varepsilon}^{(4)}, v_{\varepsilon}^{(4)}\right)\right\|_{-1} \leq C_{K} \varepsilon^{2 \widetilde{\alpha}-1-2 \kappa}
$$

and

$$
\left\|A\left(v_{\varepsilon}^{(4)}, \widetilde{\psi}_{\gamma}^{\chi}\right)\right\|_{-1} \leq C_{K} \varepsilon^{\widetilde{\alpha}-1 / 2-2 \kappa} .
$$

Fifth term. Finally, we estimate $\Lambda \Delta G\left(v_{\varepsilon}^{(3)}\right)-A\left(\widetilde{\psi}_{\gamma}^{\chi}, \widetilde{\psi}_{\gamma}^{\chi}\right)$. By definition of $\tau_{\varepsilon}^{K}$, we have $\left\|\widetilde{\psi}_{\gamma}^{\chi}\right\|_{\alpha} \leq C_{K} \varepsilon^{-\chi(\alpha-1 / 2)-\kappa}$ before time $\tau_{4}^{K}$. Since $\left\|v_{\varepsilon}^{(4)}\right\|_{\alpha} \leq$ $C_{K} \varepsilon^{-\gamma(\alpha-1 / 2)-\kappa}$ as observed before, we thus have

$$
\left\|u_{\varepsilon}^{(4)}\right\|_{\alpha} \leq C_{K} \varepsilon^{-\chi(\alpha-1 / 2)-\kappa} .
$$

Furthermore, since $\alpha>\frac{1}{2}$, there exists a constant $C>0$ such that we have the bound

$$
\begin{aligned}
\left\|\Lambda \Delta G\left(u_{\varepsilon}^{(4)}\right)-A\left(\widetilde{\psi}_{\gamma}^{\chi}, \widetilde{\psi}_{\gamma}^{\chi}\right)\right\|_{-\alpha} & =\left\|\operatorname{tr}\left(D^{2} G\left(u_{\varepsilon}^{(4)}\right)\left(\Lambda I-\Xi_{\varepsilon}\left(\widetilde{\psi}_{\gamma}^{\chi}\right)\right)\right)\right\|_{-\alpha} \\
& \leq C\left\|D^{2} G\left(u_{\varepsilon}^{(4)}\right)\right\|_{\alpha}\left\|\Lambda I-\Xi_{\varepsilon}\left(\widetilde{\psi}_{\gamma}^{\chi}\right)\right\|_{-\alpha} .
\end{aligned}
$$

Since the stopping time $\tau^{K}$ enforces that $\left\|\Lambda I-\Xi_{\varepsilon}\left(\widetilde{\psi}_{\gamma}^{\chi}\right)\right\|_{-\alpha} \leq C_{K} \varepsilon^{1 / 2}$, we infer that

$$
\left\|\Lambda \Delta G\left(u_{\varepsilon}^{(4)}\right)-A\left(\widetilde{\psi}_{\gamma}^{\chi}, \widetilde{\psi}_{\gamma}^{\chi}\right)\right\|_{-\alpha} \leq C_{K} \varepsilon^{1 / 2-\chi(\alpha-1 / 2)-\kappa} .
$$


Since $u_{\varepsilon}^{(4)}-v_{\varepsilon}^{(3)}=\varrho_{\varepsilon}+\widetilde{\psi}_{\gamma}^{\chi}$, we have by definition of $\tau^{K}$,

$$
\begin{aligned}
\left\|\Delta G\left(u_{\varepsilon}^{4}\right)-\Delta G\left(v_{\varepsilon}^{(3)}\right)\right\|_{-\alpha} & \leq\left\|\Delta G\left(u_{\varepsilon}^{4}\right)-\Delta G\left(v_{\varepsilon}^{(3)}\right)\right\|_{L^{\infty}} \\
& \leq C_{K}\left\|\varrho_{\varepsilon}\right\|_{L^{\infty}}+C_{K}\left\|\widetilde{\psi}_{\gamma}^{\chi}\right\|_{L^{\infty}} \\
& \leq C_{K}\left\|\varrho_{\varepsilon}\right\|_{\alpha}+C_{K} \varepsilon^{\gamma / 2-\kappa} .
\end{aligned}
$$

Putting these bounds together, we obtain

$$
\begin{aligned}
& \left\|\Lambda \Delta G\left(v_{\varepsilon}^{(3)}\right)-A\left(\widetilde{\psi}_{\gamma}^{\chi}, \widetilde{\psi}_{\gamma}^{\chi}\right)\right\|_{-\alpha} \\
& \quad \leq C_{K}\left(\varepsilon^{1 / 2-\chi(\alpha-1 / 2)-\kappa}+\varepsilon^{\gamma / 2-\kappa}+\left\|\varrho_{\varepsilon}\right\|_{\alpha}\right) .
\end{aligned}
$$

Sixth term. To estimate $B$, we use the fact that $\left\|u_{\varepsilon}^{(4)}(t)\right\|_{L^{\infty}} \leq C_{K}$ for $t \leq \tau_{4}^{K}$, so that one has the bound

$$
\|B\|_{L^{1}} \leq C_{K} \iint_{\mathbf{R}} \varepsilon^{2} y^{3}\left|\widehat{D}_{\varepsilon y} u_{\varepsilon}^{(4)}(x)\right|^{3}|\mu|(d y) d x .
$$

We will split this expression into two parts, using the fact that $u_{\varepsilon}^{(4)}=\widetilde{\psi}_{\gamma}^{\chi}+$ $v_{\varepsilon}^{(4)}$. First, using the fact that $\Theta\left(\widetilde{\psi}_{\gamma}^{\chi}\right) \leq C_{K} \varepsilon^{1-\kappa}$ before time $\tau_{4}^{K}$ by definition of the stopping time $\tau^{K}$, we find that

$$
\begin{aligned}
& \iint_{\mathbf{R}} \varepsilon^{2} y^{3}\left|\widehat{D}_{\varepsilon y} \widetilde{\psi}_{\gamma}^{\chi}(x)\right|^{3}|\mu|(d y) d x \\
& \quad \leq 2\left\|\widetilde{\psi}_{\gamma}^{\chi}\right\|_{L^{\infty}} \iint_{\mathbf{R}} \varepsilon y^{2}\left|\widehat{D}_{\varepsilon y} \widetilde{\psi}_{\gamma}^{\chi}(x)\right|^{2}|\mu|(d y) d x \\
& \quad=2 \varepsilon\left\|\widetilde{\psi}_{\gamma}^{\chi}\right\|_{L^{\infty}} \Theta\left(\widetilde{\psi}_{\gamma}^{\chi}\right) \leq C_{K} \varepsilon^{-2 \kappa}\left\|\widetilde{\psi}_{\gamma}^{\chi}\right\|_{L^{\infty}} \leq C_{K} \varepsilon^{\gamma / 2-3 \kappa} .
\end{aligned}
$$

Second, using the fact that $H^{1 / 6} \subseteq L^{3}$, Lemma 3.5 and the fact that $\left\|v_{\varepsilon}^{(4)}\right\|_{\alpha} \leq$ $C_{K} \varepsilon^{-\gamma(\alpha-1 / 2)-\kappa}$, we obtain

$$
\begin{aligned}
& \iint_{\mathbf{R}} \varepsilon^{2} y^{3}\left|\widehat{D}_{\varepsilon y} v_{\varepsilon}^{(4)}(x)\right|^{3}|\mu|(d y) d x \\
& \quad \leq C \varepsilon^{2} \int_{\mathbf{R}}|y|^{3}\left\|\widehat{D}_{\varepsilon y} v_{\varepsilon}^{(4)}\right\|_{1 / 6}^{3}|\mu|(d y) \\
& \quad \leq C \varepsilon^{3 \alpha-3 / 2} \int_{\mathbf{R}}|y|^{3 \alpha-1 / 2}|\mu|(d y)\left\|v_{\varepsilon}^{(4)}\right\|_{\alpha}^{3} \\
& \quad \leq C_{K} \varepsilon^{3 \widetilde{\alpha}-3 / 2-3 \kappa} .
\end{aligned}
$$

It thus follows that

$$
\|B\|_{L^{1}} \leq C_{K} \varepsilon^{\gamma / 2-3 \kappa}+C_{K} \varepsilon^{3(\widetilde{\alpha}-1 / 2)-3 \kappa} .
$$


Combining inequalities (5.9)-(5.13), we find that

$$
\begin{aligned}
\sup _{r \in(s, t)}\left\|\sigma_{\varepsilon}(r)\right\|_{-1} \leq C_{K}\left(\varepsilon^{\gamma / 2-3 \kappa}+\varepsilon^{\widetilde{\alpha}-1 / 2-2 \kappa}\right. & \\
& \left.+\varepsilon^{1 / 2-\chi(\alpha-1 / 2)-\kappa}+\sup _{r \in(s, t)}\left\|\varrho_{\varepsilon}(r)\right\|_{\alpha}\right),
\end{aligned}
$$

and the result now follows as in Proposition 5.2.

5.5. From $v_{\varepsilon}^{(4)}$ to $\widetilde{v}^{\gamma}$. Define

$$
\tau_{5}^{K}:=\tau_{4}^{K} \wedge \inf \left\{t \leq T:\left\|\widetilde{v}^{\gamma}(t)-v_{\varepsilon}^{(4)}(t)\right\|_{\alpha} \geq K\right\} .
$$

Proposition 5.7. For $\kappa>0$, we have

$$
\lim _{\varepsilon \rightarrow 0} \mathbb{P}\left(\sup _{t \leq \tau_{5}^{K}}\left\|\widetilde{v}^{\gamma}(t)-v_{\varepsilon}^{(4)}(t)\right\|_{\alpha}>\varepsilon^{1 / 2 \chi-1 / 2-\kappa}\right)=0 .
$$

Proof. Let $0 \leq s \leq t \leq \tau^{*}$. It follows from (2.4) and (2.10d) that $\varrho_{\varepsilon}:=$ $\widetilde{v}^{\gamma}-v_{\varepsilon}^{(4)}$ satisfies

where

$$
\varrho_{\varepsilon}(t)=\int_{s}^{t} S_{\varepsilon}(t-r) \sigma_{\varepsilon}(r) d r
$$

$$
\begin{aligned}
\sigma_{\varepsilon}:= & \nabla G\left(\widetilde{v}^{\gamma}+\widetilde{\psi}_{\gamma}\right) D_{\varepsilon}\left(\widetilde{v}^{\gamma}+\widetilde{\psi}_{\gamma}\right) \\
& -\nabla G\left(\widetilde{v}^{\gamma}+\widetilde{\psi}_{\gamma}^{\chi}-\varrho_{\varepsilon}\right) D_{\varepsilon}\left(\widetilde{v}^{\gamma}+\widetilde{\psi}_{\gamma}^{\chi}-\varrho_{\varepsilon}\right) \\
& +F\left(\widetilde{v}^{\gamma}+\widetilde{\psi}_{\gamma}\right)-F\left(\widetilde{v}^{\gamma}+\widetilde{\psi}_{\gamma}^{\chi}-\varrho_{\varepsilon}\right) .
\end{aligned}
$$

In order to estimate $\sigma_{\varepsilon}$, we use (5.8) to write

$$
\begin{aligned}
\sigma_{\varepsilon}= & D_{\varepsilon} G\left(\widetilde{v}^{\gamma}+\widetilde{\psi}_{\gamma}\right)-D_{\varepsilon} G\left(\widetilde{v}^{\gamma}+\widetilde{\psi}_{\gamma}^{\chi}-\varrho_{\varepsilon}\right) \\
& -\int_{\mathbf{R}} \frac{\varepsilon y^{2}}{2}\left(D^{2} G\left(u_{\varepsilon}\right)-D^{2} G\left(u_{\varepsilon}^{(4)}\right)\right)\left[\widehat{D}_{\varepsilon y} u_{\varepsilon}, \widehat{D}_{\varepsilon y} u_{\varepsilon}\right] \mu(d y) \\
& -\int_{\mathbf{R}} \frac{\varepsilon y^{2}}{2} D^{2} G\left(u_{\varepsilon}^{(4)}\right)\left[\widehat{D}_{\varepsilon y}\left(u_{\varepsilon}+u_{\varepsilon}^{(4)}\right), \widehat{D}_{\varepsilon y}\left(u_{\varepsilon}-u_{\varepsilon}^{(4)}\right)\right] \mu(d y) \\
& -\varepsilon^{2}\left(R_{\varepsilon}\left(u_{\varepsilon}, u_{\varepsilon}\right)-R_{\varepsilon}\left(u_{\varepsilon}^{(4)}, u_{\varepsilon}^{(4)}\right)\right) \\
& +F\left(\widetilde{v}^{\gamma}+\widetilde{\psi}_{\gamma}\right)-F\left(\widetilde{v}^{\gamma}+\widetilde{\psi}_{\gamma}^{\chi}-\varrho_{\varepsilon}\right) \\
= & : \sigma_{\varepsilon, 1}+\cdots+\sigma_{\varepsilon, 5},
\end{aligned}
$$

where $u_{\varepsilon}:=\widetilde{v}^{\gamma}+\widetilde{\psi}_{\gamma}, u_{\varepsilon}^{(4)}:=v_{\varepsilon}^{(4)}+\widetilde{\psi}_{\gamma}^{\chi}$ and

$$
\begin{array}{r}
R_{\varepsilon}\left(u^{1}, u^{2}\right)(x):=\int_{\mathbf{R}} \varepsilon^{2} y^{3} \int_{0}^{1} \int_{0}^{t} \int_{0}^{s} D^{3} G\left((1-r) u^{1}(x)+r u^{1}(x+\varepsilon y)\right) \\
\times\left[\widehat{D}_{\varepsilon y} u^{2}, \widehat{D}_{\varepsilon y} u^{2}, \widehat{D}_{\varepsilon y} u^{2}\right] d r d s d t d \mu(y) .
\end{array}
$$

We shall now estimate $\sigma_{\varepsilon, 1}, \ldots, \sigma_{\varepsilon, 5}$ individually. 
First term. First, we observe that $\widetilde{v}^{\gamma}, \widetilde{\psi}_{\gamma}, \widetilde{\psi}_{\gamma}^{\chi}$ and $\varrho_{\varepsilon}$ are bounded in $L^{\infty}$ before time $\tau_{5}^{K}$. Using Lemma 3.5, the embedding $H^{\alpha} \subseteq L^{\infty}$, and the definition of the stopping time to bound $\left\|\widetilde{\psi}_{\chi}\right\|_{L^{\infty}}$, we obtain

$$
\begin{aligned}
\left\|\sigma_{\varepsilon, 1}\right\|_{-1} & =\left\|D_{\varepsilon}\left(G\left(\widetilde{v}^{\gamma}+\widetilde{\psi}_{\gamma}\right)-G\left(\widetilde{v}^{\gamma}+\widetilde{\psi}_{\gamma}^{\chi}-\varrho_{\varepsilon}\right)\right)\right\|_{-1} \\
& \leq C\left\|G\left(\widetilde{v}^{\gamma}+\widetilde{\psi}_{\gamma}\right)-G\left(\widetilde{v}^{\gamma}+\widetilde{\psi}_{\gamma}^{\chi}-\varrho_{\varepsilon}\right)\right\|_{L^{\infty}} \\
& \leq C_{K}\left(\left\|\widetilde{\psi}_{\chi}\right\|_{L^{\infty}}+\left\|\varrho_{\varepsilon}\right\|_{L^{\infty}}\right) \\
& \leq C_{K}\left(\varepsilon^{\chi / 2-\kappa}+\left\|\varrho_{\varepsilon}\right\|_{\alpha}\right) .
\end{aligned}
$$

Second term. Using Lemma 3.5 and the fact that $\varepsilon^{\gamma(\alpha-1 / 2)+\kappa}\left\|\widetilde{v}^{\gamma}\right\|_{\alpha}$ is bounded before time $\tau_{5}^{K}$, we estimate

$$
\begin{aligned}
\Theta_{\varepsilon}\left(\widetilde{v}^{\gamma}\right) & =\int_{\mathbf{R}} y^{2}\left\|\widehat{D}_{\varepsilon y} \widetilde{v}^{\gamma}\right\|_{L^{2}}^{2}|\mu|(d y) \\
& \leq C_{K} \int_{\mathbf{R}} y^{2}|\varepsilon y|^{2(\alpha-1)}\left\|\widetilde{v}^{\gamma}\right\|_{\alpha}^{2}|\mu|(d y) \\
& \leq C_{K} \int_{\mathbf{R}} y^{2}|\varepsilon y|^{2(\alpha-1)} \varepsilon^{-2 \gamma(\alpha-1 / 2)-2 \kappa}|\mu|(d y) \\
& \leq C_{K} \varepsilon^{2 \widetilde{\alpha}-2-\kappa}
\end{aligned}
$$

and by the definition of the stopping time $\tau^{K}$,

$$
\Theta_{\varepsilon}\left(\widetilde{\psi}_{\gamma}\right) \leq C_{K} \varepsilon^{-1-\kappa}
$$

As a consequence,

$$
\Theta_{\varepsilon}\left(u_{\varepsilon}\right) \leq 2\left(\Theta_{\varepsilon}\left(\widetilde{v}^{\gamma}\right)+\Theta_{\varepsilon}\left(\widetilde{\psi}_{\gamma}\right)\right) \leq C_{K}\left(\varepsilon^{2 \widetilde{\alpha}-2-\kappa}+\varepsilon^{-1-\kappa}\right) \leq \varepsilon^{-1-\kappa} .
$$

Note that $\left\|u_{\varepsilon}\right\|_{L^{\infty}}$ and $\left\|u_{\varepsilon}^{(4)}\right\|_{L^{\infty}}$ are bounded before time $\tau_{5}^{K}$. Using that $L^{1} \subseteq H^{-1}$, we obtain

$$
\begin{aligned}
\left\|\sigma_{\varepsilon, 2}\right\|_{-1} & \leq\left\|\sigma_{\varepsilon, 2}\right\|_{L^{1}} \leq \varepsilon\left\|D^{2} G\left(u_{\varepsilon}\right)-D^{2} G\left(u_{\varepsilon}^{(4)}\right)\right\|_{L^{\infty}} \Theta_{\varepsilon}\left(u_{\varepsilon}\right) \\
& \leq C_{K} \varepsilon^{-2 \kappa}\left\|u_{\varepsilon}-u_{\varepsilon}^{(4)}\right\|_{L^{\infty}} \\
& \leq C_{K} \varepsilon^{-2 \kappa}\left(\left\|\varrho_{\varepsilon}\right\|_{L^{\infty}}+\left\|\widetilde{\psi}_{\chi}\right\|_{L^{\infty}}\right) \\
& \leq C_{K} \varepsilon^{-2 \kappa}\left(\left\|\varrho_{\varepsilon}\right\|_{\alpha}+\varepsilon^{\chi / 2-\kappa}\right) .
\end{aligned}
$$

Third term. By Lemma 3.5, we have

$$
\begin{aligned}
\Theta_{\varepsilon}\left(\varrho_{\varepsilon}\right) & =\int_{\mathbf{R}} y^{2}\left\|\widehat{D}_{\varepsilon y} \varrho_{\varepsilon}\right\|_{L^{2}}^{2}|\mu|(d y) \\
& \leq C \int_{\mathbf{R}} y^{2}|\varepsilon y|^{2(\alpha-1)}\left\|\varrho_{\varepsilon}\right\|_{\alpha}^{2}|\mu|(d y) \leq C \varepsilon^{2 \alpha-2}\left\|\varrho_{\varepsilon}\right\|_{\alpha}^{2} .
\end{aligned}
$$


Observe that $u_{\varepsilon}+u_{\varepsilon}^{(4)}=2 \widetilde{v}^{\gamma}-\varrho_{\varepsilon}+\widetilde{\psi}_{\gamma}+\widetilde{\psi}_{\gamma}^{\chi}$ and $u_{\varepsilon}-u_{\varepsilon}^{(4)}=\widetilde{\psi}_{\chi}+\varrho_{\varepsilon}$. Taking into account that

$$
\varepsilon^{1+\kappa} \Theta_{\varepsilon}\left(\widetilde{\psi}_{\gamma}\right), \quad \varepsilon^{1+\kappa} \Theta_{\varepsilon}\left(\widetilde{\psi}_{\gamma}\right), \quad \varepsilon^{2-\chi+\kappa} \Theta_{\varepsilon}\left(\widetilde{\psi}_{\chi}\right), \quad\left\|\varrho_{\varepsilon}\right\|_{\alpha}
$$

are all bounded before time $\tau_{5}^{K}$, we obtain

$$
\begin{aligned}
\Theta_{\varepsilon}\left(u_{\varepsilon}+u_{\varepsilon}^{(4)}\right) & \leq C\left(\Theta_{\varepsilon}\left(\widetilde{v}^{\gamma}\right)+\Theta_{\varepsilon}\left(\varrho_{\varepsilon}\right)+\Theta_{\varepsilon}\left(\widetilde{\psi}_{\gamma}\right)+\Theta_{\varepsilon}\left(\widetilde{\psi}_{\gamma}^{\chi}\right)\right) \\
& \leq C_{K}\left(\varepsilon^{2 \widetilde{\alpha}-2-\kappa}+\varepsilon^{2 \alpha-1}+\varepsilon^{-1-\kappa}\right) \leq C_{K} \varepsilon^{-1-\kappa}
\end{aligned}
$$

and

$$
\begin{aligned}
\Theta_{\varepsilon}\left(u_{\varepsilon}-u_{\varepsilon}^{(4)}\right) & \leq C\left(\Theta_{\varepsilon}\left(\widetilde{\psi}_{\chi}\right)+\Theta_{\varepsilon}\left(\varrho_{\varepsilon}\right)\right) \\
& \leq C_{K} \varepsilon^{\chi-2-\kappa}+C \varepsilon^{2 \alpha-2}\left\|\varrho_{\varepsilon}\right\|_{\alpha}^{2} .
\end{aligned}
$$

Using that $\left\|u_{\varepsilon}^{(4)}\right\|_{L^{\infty}} \leq C_{K}$ before time $\tau_{5}^{K}$, we obtain

$$
\begin{aligned}
\left\|\sigma_{\varepsilon, 3}\right\|_{-1} & \leq\left\|\sigma_{\varepsilon, 3}\right\|_{L^{1}} \leq \varepsilon\left\|D^{2} G\left(u_{\varepsilon}^{(4)}\right)\right\|_{L^{\infty}} \sqrt{\Theta_{\varepsilon}\left(u_{\varepsilon}+u_{\varepsilon}^{(4)}\right) \Theta_{\varepsilon}\left(u_{\varepsilon}-u_{\varepsilon}^{(4)}\right)} \\
& \leq C_{K}\left(\varepsilon^{\chi / 2-1 / 2-2 \kappa}+\varepsilon^{\alpha-1 / 2-\kappa}\left\|\varrho_{\varepsilon}\right\|_{\alpha}\right) .
\end{aligned}
$$

Fourth term. We shall show that

$$
\left\|\sigma_{\varepsilon, 4}\right\|_{-1} \leq C_{K}\left(\varepsilon^{-2 \kappa}\left\|\varrho_{\varepsilon}\right\|_{\alpha}+\varepsilon^{\chi / 2-1 / 2-2 \kappa}\right) .
$$

First, we use the $L^{\infty}$-bound on $u_{\varepsilon}^{(4)}$ enforced by the stopping time, to obtain the pointwise bound

$$
\begin{aligned}
& \mid R_{\varepsilon}\left(u_{\varepsilon}, u_{\varepsilon}\right)- R_{\varepsilon}\left(u_{\varepsilon}, u_{\varepsilon}^{(4)}\right) \mid \\
& \leq C_{K} \int_{\mathbf{R}} \varepsilon^{2} y^{3}\left(\left|\widehat{D}_{\varepsilon y} u_{\varepsilon}\right|^{2}+\left|\widehat{D}_{\varepsilon y} u_{\varepsilon}\right|\left|\widehat{D}_{\varepsilon y} u_{\varepsilon}^{(4)}\right|+\left|\widehat{D}_{\varepsilon y} u_{\varepsilon}^{(4)}\right|^{2}\right) \\
& \quad \quad \times\left|\widehat{D}_{\varepsilon y}\left(u_{\varepsilon}-u_{\varepsilon}^{(4)}\right)\right||\mu|(d y) \\
& \leq C_{K} \int_{\mathbf{R}} \varepsilon y^{2}\left(\left|\widehat{D}_{\varepsilon y} u_{\varepsilon}\right|+\left|\widehat{D}_{\varepsilon y} u_{\varepsilon}^{(4)}\right|\right)\left|\widehat{D}_{\varepsilon y}\left(u_{\varepsilon}-u_{\varepsilon}^{(4)}\right)\right||\mu|(d y) .
\end{aligned}
$$

In view of (5.20) it thus follows that

$$
\begin{aligned}
& \left\|R_{\varepsilon}\left(u_{\varepsilon}, u_{\varepsilon}\right)-R_{\varepsilon}\left(u_{\varepsilon}, u_{\varepsilon}^{(4)}\right)\right\|_{L^{1}} \\
& \quad \leq C_{K} \varepsilon \int_{\mathbf{R}} y^{2}\left\|\left(\left|\widehat{D}_{\varepsilon y} u_{\varepsilon}\right|+\left|\widehat{D}_{\varepsilon y} u_{\varepsilon}^{(4)}\right|\right)\left|\widehat{D}_{\varepsilon y}\left(u_{\varepsilon}-u_{\varepsilon}^{(4)}\right)\right|\right\|_{L^{1}}|\mu|(d y) \\
& \quad \leq C_{K} \varepsilon \int_{\mathbf{R}} y^{2}\left(\left\|\widehat{D}_{\varepsilon y} u_{\varepsilon}\right\|_{L^{2}}+\left\|\widehat{D}_{\varepsilon y} u_{\varepsilon}^{(4)}\right\|_{L^{2}}\right)\left\|\widehat{D}_{\varepsilon y}\left(u_{\varepsilon}-u_{\varepsilon}^{(4)}\right)\right\|_{L^{2}}|\mu|(d y) \\
& \quad \leq C_{K} \varepsilon \sqrt{\left(\Theta_{\varepsilon}\left(u_{\varepsilon}\right)+\Theta_{\varepsilon}\left(u_{\varepsilon}^{(4)}\right)\right) \Theta_{\varepsilon}\left(u_{\varepsilon}-u_{\varepsilon}^{(4)}\right)} .
\end{aligned}
$$


Using $(5.16),(5.18)$, and the definition of the stopping time to bound $\Theta_{\varepsilon}\left(\widetilde{\psi}_{\chi}\right)$, we find that

$$
\begin{aligned}
\Theta_{\varepsilon}\left(u_{\varepsilon}\right)+\Theta_{\varepsilon}\left(u_{\varepsilon}^{(4)}\right) & \leq C\left(\Theta_{\varepsilon}\left(u_{\varepsilon}\right)+\Theta_{\varepsilon}\left(\varrho_{\varepsilon}\right)+\Theta_{\varepsilon}\left(\widetilde{\psi}_{\chi}\right)\right) \\
& \leq C_{K}\left(\varepsilon^{-1-\kappa}+\varepsilon^{2 \alpha-2}+\varepsilon^{\chi-1-\kappa}\right) \leq \varepsilon^{-1-\kappa} .
\end{aligned}
$$

Using (5.20), we thus obtain

$$
\left\|R_{\varepsilon}\left(u_{\varepsilon}, u_{\varepsilon}\right)-R_{\varepsilon}\left(u_{\varepsilon}, u_{\varepsilon}^{(4)}\right)\right\|_{L^{1}} \leq C_{K}\left(\varepsilon^{\chi / 2-1 / 2-2 \kappa}+\varepsilon^{\alpha-1 / 2-\kappa}\left\|\varrho_{\varepsilon}\right\|_{\alpha}\right) .
$$

Furthermore, taking into account that

$$
\left\|u_{\varepsilon}-u_{\varepsilon}^{(4)}\right\|_{L^{\infty}} \leq C_{K}\left(\left\|\widetilde{\psi}_{\chi}\right\|_{L^{\infty}}+\left\|\varrho_{\varepsilon}\right\|_{L^{\infty}}\right) \leq C_{K}\left(\varepsilon^{\chi / 2-\kappa}+\left\|\varrho_{\varepsilon}\right\|_{\alpha}\right),
$$

we have by (5.16),

$$
\begin{aligned}
& \left\|R_{\varepsilon}\left(u_{\varepsilon}, u_{\varepsilon}^{(4)}\right)-R_{\varepsilon}\left(u_{\varepsilon}^{(4)}, u_{\varepsilon}^{(4)}\right)\right\|_{L^{1}} \\
& \quad \leq C_{K} \varepsilon^{2}\left\|u_{\varepsilon}-u_{\varepsilon}^{(4)}\right\|_{L^{\infty}} \int_{\mathbf{R}} y^{3}\left\|\left|\widehat{D}_{\varepsilon y} u_{\varepsilon}^{(4)}\right|^{3}\right\|_{L^{1}}|\mu|(d y) \\
& \quad \leq C_{K} \varepsilon\left\|u_{\varepsilon}-u_{\varepsilon}^{(4)}\right\|_{L^{\infty}} \int_{\mathbf{R}} y^{2}\left\|\widehat{D}_{\varepsilon y} u_{\varepsilon}^{(4)}\right\|_{L^{2}}^{2}|\mu|(d y) \\
& \quad=C_{K} \varepsilon\left\|u_{\varepsilon}-u_{\varepsilon}^{(4)}\right\|_{L^{\infty}} \Theta_{\varepsilon}^{2}\left(u_{\varepsilon}^{(4)}\right) \\
& \quad \leq C_{K}\left(\varepsilon^{\chi / 2-3 \kappa}+\varepsilon^{-2 \kappa}\left\|\varrho_{\varepsilon}\right\|_{\alpha}\right) .
\end{aligned}
$$

The claim follows by adding (5.22) and (5.23) and using the embedding $L^{1} \subseteq H^{-1}$.

Fifth term. As in the first step, we have

$$
\begin{aligned}
\left\|\sigma_{\varepsilon, 4}\right\|_{-1} & =\left\|F\left(\widetilde{v}^{\gamma}+\widetilde{\psi}_{\gamma}\right)-F\left(\widetilde{v}^{\gamma}+\widetilde{\psi}_{\gamma}^{\chi}-\varrho_{\varepsilon}\right)\right\|_{-1} \\
& \leq C_{K}\left(\left\|\widetilde{\psi}_{\chi}\right\|_{L^{\infty}}+\left\|\varrho_{\varepsilon}\right\|_{L^{\infty}}\right) \\
& \leq C_{K}\left(\varepsilon^{\chi / 2-\kappa}+\left\|\varrho_{\varepsilon}\right\|_{\alpha}\right) .
\end{aligned}
$$

Combining the five estimates, we obtain

$$
\begin{aligned}
\left\|\varrho_{\varepsilon}(t)\right\|_{\alpha} & \leq C(t-s)^{(1-\alpha) / 2} \sup _{r \in(s, t)}\left\|\sigma_{\varepsilon}(r)\right\|_{-1} \\
& \leq C(t-s)^{(1-\alpha) / 2} \sup _{r \in(s, t)}\left(\varepsilon^{-2 \kappa}\left\|\varrho_{\varepsilon}(r)\right\|_{\alpha}+\varepsilon^{\chi / 2-1 / 2-2 \kappa}\right) .
\end{aligned}
$$

The result now follows as in the proof of Proposition 5.1.

Acknowledgments. We are grateful to Hendrik Weber, Jochen Voß and Andrew Stuart for numerous discussions on this and related problems. We thank the anonymous referee for useful comments. Part of this work has been carried out while J. Maas was visiting the Courant Institute and the 
University of Warwick. He thanks both institutions for their kind hospitality and support.

\section{REFERENCES}

1] Adams, R. A. and Fournier, J. J. F. (2003). Sobolev Spaces, 2nd ed. Pure and Applied Mathematics (Amsterdam) 140. Elsevier, Amsterdam. MR2424078

[2] Bertini, L., Cancrini, N. and Jona-Lasinio, G. (1994). The stochastic Burgers equation. Comm. Math. Phys. 165 211-232. MR1301846

[3] Bertini, L. and Giacomin, G. (1997). Stochastic Burgers and KPZ equations from particle systems. Comm. Math. Phys. 183 571-607. MR1462228

[4] Brzeźniak, Z., Capiński, M. and Flandoli, F. (1991). Stochastic partial differential equations and turbulence. Math. Models Methods Appl. Sci. 1 41-59. MR1105007

[5] Courant, R., Isafacson, E. and Rees, M. (1952). On the solution of nonlinear hyperbolic differential equations by finite differences. Comm. Pure Appl. Math. 5 243-255. MR0053336

[6] Da Prato, G., Debussche, A. and Temam, R. (1994). Stochastic Burgers' equation. NoDEA Nonlinear Differential Equations Appl. 1 389-402. MR1300149

[7] Da Prato, G. and Zabczyk, J. (1992). Stochastic Equations in Infinite Dimensions. Encyclopedia of Mathematics and Its Applications 44. Cambridge Univ. Press, Cambridge. MR1207136

[8] DeVore, R. A. and Sharpley, R. C. (1993). Besov spaces on domains in $\mathbf{R}^{d}$. Trans. Amer. Math. Soc. 335 843-864. MR1152321

[9] GyÖngy, I. (1998). Existence and uniqueness results for semilinear stochastic partial differential equations. Stochastic Process. Appl. 73 271-299. MR1608641

[10] Hairer, M. (2009). An introduction to stochastic PDEs. Available at arXiv: 0907.4178.

[11] Hairer, M. (2011). Singular perturbations to semilinear stochastic heat equations. Probab. Theory Related Fields. To appear. Available at arXiv:1002.3722.

[12] Hairer, M. and Voss, J. (2010). Approximations to the stochastic Burgers equation. J. Nonlinear Sci. To appear. Available at arXiv:1005.4438.

[13] Kwapié, S. and Woyczyński, W. A. (1992). Random Series and Stochastic Integrals: Single and Multiple. Birkhäuser, Boston, MA. MR1167198

[14] Lunardi, A. (1995). Analytic Semigroups and Optimal Regularity in Parabolic Problems. Progress in Nonlinear Differential Equations and Their Applications 16. Birkhäuser, Basel. MR1329547

[15] Mattheij, R. M. M., Rienstra, S. W. and ten Thije Boonkkamp, J. H. M. (2005). Partial Differential Equations: Modeling, Analysis, Computation. SIAM, Philadelphia, PA. MR2178809

[16] Revuz, D. and Yor, M. (1994). Continuous Martingales and Brownian Motion, 2nd ed. Grundlehren der Mathematischen Wissenschaften [Fundamental Principles of Mathematical Sciences] 293. Springer, Berlin. MR1303781

[17] Wong, E. and ZAKAI, M. (1965). On the convergence of ordinary integrals to stochastic integrals. Ann. Math. Statist. 36 1560-1564. MR0195142

Department of Mathematics

UNIVERSITY OF WARWICK

COVEnTRY CV4 7AL

United KingDom

E-MAIL: M.Hairer@Warwick.ac.uk
Institute For APplied Mathematics UNIVERSITY OF BONN

ENDENiCher Allee 60

53115 BONN

Germany

E-MAIL: maas@iam.uni-bonn.de 